\title{
Tau pathology mediates the effects of amyloid on neocortical tau propagation and neurodegeneration among individuals without dementia
}

\author{
Murat Bilgel ${ }^{\text {f }}$, Dean F. Wong ${ }^{2}$, Abhay R. Moghekar ${ }^{3}$, Luigi Ferrucci ${ }^{4}$, Susan M. Resnick ${ }^{1}$, and for the Alzheimer's \\ Disease Neuroimaging Initiative' \\ ${ }^{1}$ Brain Aging and Behavior Section, Laboratory of Behavioral Neuroscience, National Institute on Aging, Baltimore, \\ Maryland, United States \\ ${ }^{2}$ Mallinckrodt Institute of Radiology, Washington University School of Medicine, St. Louis, Missouri, United States \\ ${ }^{3}$ Department of Neurology, Johns Hopkins University School of Medicine, Baltimore, Maryland, United States \\ ${ }^{4}$ Longitudinal Studies Section, Translational Gerontology Branch, National Institute on Aging, Baltimore, Maryland, \\ United States
}

\begin{abstract}
Amyloid is associated with greater tau pathology and is thought to facilitate tau propagation from medial temporal lobe (MTL) to the neocortex, where tau is closely associated with local neurodegeneration. We used causal mediation analysis to quantify the involvement of amyloid and tau in tau propagation and neurodegeneration, as measured by cerebral blood flow, glucose metabolism, and volume, in two longitudinal samples of individuals without dementia. Greater entorhinal tau was associated with greater inferior temporal tau. This association was stronger among amyloid+ individuals, suggesting that amyloid facilitates tau propagation from MTL to neocortex. Entorhinal tau mediated up to $50 \%$ of the total effect of amyloid on inferior temporal tau. Higher inferior temporal tau was associated with colocalized neurodegeneration, including lower cerebral blood flow, glucose metabolism, and regional volume, whereas amyloid had only an indirect effect on neurodegeneration via tau, implying that tau is the primary driver of neurodegeneration. Our findings suggest targeting amyloid or MTL tau might slow down neocortical spread of tau, but a combination therapy may yield better outcomes.
\end{abstract}

\section{Introduction}

In post mortem studies, hyperphosphorylated tau protein aggregates are found universally in the medial temporal lobe (MTL) among individuals over the age of 70 regardless of cognitive status (Nelson et al., 2012). Among cognitively unimpaired individuals, the prevalence of temporal lobe tau pathology increases with age (Jack et al., 2017). Tau pathology spreads from the MTL to the neocortex primarily via synaptic connections (Calafate et al. 2015, De Calignon et al. 2012, Liu et al., 2012, Wu et al., 2016). While tau pathology can occur in the absence of amyloid plaques, amyloid increases the likelihood of the development of tau pathology. Soluble amyloid- $\beta$ (A $\beta$ ) oligomers have been demonstrated to induce tau hyperphosphorylation in hippocampal neurons in the absence of fibrillar $\mathrm{A} \beta$ (Jin) et al., 2011). Amyloid pathology also facilitates trans-synaptic tau transmission (Pooler et al., 2015). A recent longitudinal study of participants who were cognitively normal or had varying degrees of clinical impairment found that levels of amyloid and MTL tau are independently associated with greater rates of change in inferior temporal gyrus (ITG) tau, and that an interaction between rhinal cortex tau and amyloid load accounts for about $35 \%$ of the variance in the rate of change in ITG tau (Sanchez et al., 2021), providing evidence that amyloid accelerates the propagation of tau. In agreement with this finding, several observational studies found that baseline $\mathrm{A} \beta$ levels are associated with faster tau accumulation over time (Buckley et al., 2019, Jack et al., 2018).

Once tau pathology spreads to the neocortex, it becomes closely linked with neurodegeneration and cognitive symptoms. Neocortical tau pathology is observed in several neurodegenerative diseases, with Alzheimer's disease

\footnotetext{
*Corresponding author: murat.bilgel@nih.gov

$\dagger$ Data used in preparation of this article were obtained from the Alzheimer's Disease Neuroimaging Initiative (ADNI) database (adni.loni.usc.edu). As such, the investigators within the ADNI contributed to the design and implementation of ADNI and/or provided data but did not participate in analysis or writing of this report. A complete listing of ADNI investigators can be found at: http://adni.loni.usc.edu/wp-content/uploads/how_to_apply/ADNI_Acknowledgement_List.pdf
} 
(AD) being the most prevalent tauopathy. The spatial distribution of cortical tau is distinct across different clinical syndromes (Ossenkoppele et al., 2016) and overlaps that of local atrophy (La Joie et al., 2020). Tau pathology is also associated locally with other markers of neurodegeneration, including lower cerebral blood flow (CBF) (Albrecht et al., 2020; Rubinski et al., 2021), glucose metabolism (Hanseeuw et al., 2017; Strom et al., 2021), and volume (Iaccarino et al. 2018). However, the extent of neurodegeneration is greater than what is accounted for by tau pathology (Gómez-Isla et al., 1997), suggesting that additional factors are involved. One such factor is thought to be amyloid pathology. Studies have found associations between amyloid pathology and greater atrophy among cognitively normal individuals (Chételat et al. 2010), lower glucose metabolism among individuals without dementia (Carbonell et al., 2020, Teipel and Grothe, 2016) and among cognitively normal individuals (Petersen et al. 2016), lower CBF in a sample consisting mostly of individuals without dementia (Rubinski et al., 2021), and lower hippocampal volumes among cognitively normal individuals (Petersen et al., 2016: Villemagne et al., 2013). Additionally, one study found an interaction between amyloid and tau in relation to glucose metabolism such that greater amyloid burden was linked to a stronger association between tau and hypometabolism among patients with clinically diagnosed AD (Bischof et al. 2016$)$.

Given the potential role of neocortical tau in early neurodegeneration and cognitive decline, it is important to prevent its spread from the MTL. Prior literature, largely based on samples including participants who have cognitive impairment or dementia as well as those who are cognitively normal, provides strong evidence for the involvement of both amyloid pathology and MTL tau in propagating tau to the neocortex, and for the involvement of both amyloid and tau in neurodegeneration. However, the extent to which amyloid versus existing tau pathology is involved in propagating tau to the neocortex and in exerting neurodegenerative effects among individuals without dementia remains unclear, especially considering that not all longitudinal studies of cognitively normal older adults have found an association between amyloid and rate of tau accumulation (Harrison et al., 2018). If the propagation of tau and neurodegeneration is mainly driven by existing tau pathology, then interventions to alleviate amyloid pathology would not be expected to prevent the cortical spreading of tau or neurodegenerative changes. On the other hand, if amyloid has a substantial direct effect in the neocortical propagation of tau or neurodegeneration, then successful therapies would need to target both neuropathologies simultaneously. Quantifying the degree of involvement of amyloid and tau pathology in the propagation of tau and neurodegeneration will inform strategies to prevent or delay the onset of clinical symptoms.

In this study, we focus on three scientific questions: (i) Does amyloid facilitate the spread of tau from MTL to the neocortex? (ii) To what extent is neocortical tau accumulation driven by amyloid versus MTL tau pathology? (iii) To what extent is neuronal activity, metabolism, or regional volume loss driven by amyloid versus local tau pathology? We investigate these associations among individuals without dementia in two distinct longitudinal studies to gain insight into changes that occur early in the disease process. To assess if amyloid facilitates the spread of tau from the MTL to the neocortex, we investigate whether amyloid moderates the cross-sectional association between tau in the entorhinal cortex (EC) and the ITG, which is an early neocortical region exhibiting tau pathology (Berron et al., 2021). To quantify the involvement of amyloid versus MTL tau in propagating tau, we evaluate whether EC tau mediates the association between amyloid and ITG tau using causal mediation analysis, which allows us to take into account the moderating effect of amyloid on tau propagation. We investigate the local relationships between amyloid and neurodegeneration in the ITG, as measured by CBF, glucose metabolism, and regional volume, and whether these relationships are mediated by ITG tau. Finally, we use longitudinal data to examine the associations of amyloid and ITG tau with rates of change in measures of ITG neurodegeneration to assess the directional assumptions of our causal models.

\section{Methods}

\subsection{Participants}

We investigated our scientific questions in two distinct longitudinal studies: the BLSA was the primary data set used for this study, and we repeated our statistical analyses using the ADNI data set (adni.Ioni.usc.edu)

\footnotetext{
$\ddagger$ The ADNI was launched in 2003 as a public-private partnership, led by Principal Investigator Michael W. Weiner, MD. The primary goal of ADNI has been to test whether serial magnetic resonance imaging (MRI), positron emission tomography (PET), other biological markers, and clinical and neuropsychological assessment can be combined to measure the progression of mild cognitive impairment (MCI) and early AD.
} 
The study samples included participants without dementia who had apolipoprotein E ( $A P O E$ ) genotyping, at least one ${ }^{18} \mathrm{~F}$-flortaucipir (FTP) positron emission tomography (PET) scan, as well as a $T_{1}$-weighted magnetic resonance imaging (MRI) and an amyloid PET scan within 3.5 years of their baseline FTP PET scan. The radiotracer used for amyloid PET was ${ }^{11} \mathrm{C}$-Pittsburgh compound B (PiB) in the BLSA, and ${ }^{18} \mathrm{~F}$-florbetapir (FBP) in the ADNI. Dynamic amyloid PET acquisition in the BLSA allowed for the computation of the relative radiotracer delivery parameter $R_{1}$, which is a surrogate measure of $\mathrm{CBF}$ (Bilgel et al. 2019). In the ADNI, instead of CBF, we used a measure of glucose metabolism as assessed by ${ }^{18} \mathrm{~F}$-fluorodeoxyglucose (FDG) PET $\$$. Both CBF and glucose metabolism are correlates of neuronal activity (Sokoloff, 1993). ADNI participants without an FDG PET scan within 3.5 years of their baseline FTP PET or of the corresponding amyloid PET scans were excluded from the study sample.

Cross-sectional analyses were based on measurements acquired at (or within 3.5 years of) the baseline FTP PET visit. For longitudinal analyses investigating the rate of change in PET measurements, we included visits within \pm 5 years of baseline FTP PET. Participants were non-demented at all visits included in the longitudinal analyses.

In the BLSA, cognitively normal (CN) status was based on either (i) a Clinical Dementia Rating (Morris, 1993) of zero and $\leq 3$ errors on the Blessed Information-Memory-Concentration Test (Fuld, 1978), and therefore the participant did not meet criteria for consensus conference; or (ii) the participant met criteria for consensus conference and was determined to be $\mathrm{CN}$ based on thorough review of clinical and neuropsychological data. Mild cognitive impairment (MCI) diagnoses were determined according to Petersen criteria (Petersen, 2004). At enrollment into the PET neuroimaging substudy of the BLSA, all participants were free of central nervous system disease (dementia, stroke, bipolar illness, epilepsy), severe cardiac disease, severe pulmonary disease, and metastatic cancer. The assessment of cognitive status and enrollment criteria in the ADNI are described elsewhere (Petersen et al., 2010).

The BLSA research protocols were conducted in accordance with United States federal policy for the protection of human research subjects contained in Title 45 Part 46 of the Code of Federal Regulations (45 CFR 46), approved by local institutional review boards (IRB), and all participants gave written informed consent at each visit. The BLSA PET substudy is governed by the Johns Hopkins Medical Institutions IRB, and the BLSA study is overseen by the National Institute of Environmental Health Sciences IRB. The ADNI study was approved by the IRBs of all of the participating institutions, and all participants gave written informed consent.

\subsection{MR imaging}

\subsubsection{BLSA}

Magnetization-prepared rapid gradient echo (MPRAGE) scans were acquired on a $3 \mathrm{~T}$ Philips Achieva scanner (TR $=6.8 \mathrm{~ms}, \mathrm{TE}=3.2 \mathrm{~ms}$, flip angle $=8^{\circ}$, image matrix $=256 \times 256,170$ slices, voxel size $=1 \mathrm{~mm} \times 1 \mathrm{~mm} \times 1.2 \mathrm{~mm}$ ). We computed anatomical labels and regional brain volumes using Multi-atlas region Segmentation using Ensembles of registration algorithms and parameters (MUSE) (Doshi et al., 2016), and intracranial volume (ICV) was determined from intracranial masks computed using a deep learning approach (Doshi et al., 2019). We computed ICV-adjusted residuals for ITG volume using the approach described by (Jack et al., 1989).

\subsubsection{ADNI}

For a detailed description of MRI protocols, see adni.loni.usc.edu. We used tabular data provided by the ADNI MRI Core at the University of California San Francisco for regional volumes. These were obtained with cross-sectional FreeSurfer 6.0 (https://surfer.nmr.mgh.harvard.edu/) processing of MPRAGE/IRSPGR scans based on the Desikan-Killiany atlas (Desikan et al., 2006). We computed ICV-adjusted residuals for ITG volume as described for the BLSA.

\subsection{PET imaging}

\subsubsection{BLSA}

FTP PET scans were obtained over 30 min on a Siemens High Resolution Research Tomograph (HRRT) scanner starting $75 \mathrm{~min}$ after an intravenous bolus injection of approximately $370 \mathrm{MBq}$ of radiotracer. Dynamic images were

\footnotetext{
$\S$ While the ADNI study includes ASL-MRI to quantify CBF, our inclusion criteria resulted in a very small sample for the ASL-MRI measures. Because of this, we chose to use FDG PET measures instead.
} 
reconstructed using ordered subset expectation-maximization to yield 6 time frames of 5 min each with approximately $2.5 \mathrm{~mm}$ full width at half maximum $(\mathrm{FWHM})$ at the center of the field of view (image matrix $=256 \times 256,207$ slices, voxel size $=1.22 \mathrm{~mm} \times 1.22 \mathrm{~mm} \times 1.22 \mathrm{~mm}$ ). Following time frame alignment, the $80 \mathrm{~min}$ to 100 min average PET image was partial volume corrected using the region-based voxelwise method (Thomas et al., 2011). The corrected image was then used to compute standardized uptake value ratio (SUVR) images using the inferior cerebellar gray matter as the reference region. FTP PET image analysis workflow is described in more detail in Ziontz et al. (2019). We computed the average bilateral SUVR in the EC and the ITG.

PiB PET scans were obtained over 70 min on either a GE Advance (181 scans) or a Siemens HRRT (96 scans) scanner immediately following an intravenous bolus injection of approximately $555 \mathrm{MBq}$ of radiotracer. Scans acquired on the GE Advance were reconstructed using filtered backprojection with a ramp filter to yield 33 time frames with approximately $4.5 \mathrm{~mm}$ FWHM at the center of the field of view (image matrix $=128 \times 128$, 35 slices, voxel size $=2 \mathrm{~mm} \times 2 \mathrm{~mm} \times 4.25 \mathrm{~mm}$ ). Scans acquired on the Siemens HRRT were reconstructed using ordered subset expectation-maximization to yield 33 time frames with approximately $2.5 \mathrm{~mm}$ FWHM at the center of the field of view (image matrix $=256 \times 256,207$ slices, voxel size $=1.22 \mathrm{~mm} \times 1.22 \mathrm{~mm} \times 1.22 \mathrm{~mm}$ ). Since most of the PiB PET scans were acquired on the GE Advance, the reconstructed HRRT scans were smoothed with a $3 \mathrm{~mm}$ FWHM isotropic Gaussian kernel to bring their spatial resolution closer to that of the GE Advance scans, and then resampled to match the voxel size of the GE Advance PiB PET scans. Following time frame alignment and co-registration with MRI, distribution volume ratio (DVR) and relative radiotracer delivery $\left(R_{1}\right)$ images were computed using a spatially constrained simplified reference tissue model with cerebellar gray matter as the reference region (Zhou et al. 2007). Mean cortical amyloid burden was calculated as the average of the DVR values in cingulate, frontal, parietal (including precuneus), lateral temporal, and lateral occipital cortical regions, excluding the sensorimotor strip. Leveraging longitudinal PiB PET data available on both GE Advance and HRRT scanners for 79 BLSA participants, we estimated the parameters of a linear model mapping mean cortical DVR values between the GE Advance and HRRT scanners, and applied this mapping to all HRRT values to harmonize them with the GE Advance values. Individuals were categorized as amyloid $-/+$ based on a mean cortical DVR threshold of 1.06, which was derived from a Gaussian mixture model fitted to harmonized mean cortical DVR values at baseline. PiB PET image analysis workflow is described in further detail in Bilgel et al. (2019).

\subsubsection{ADNI}

For a detailed description of PET protocols, see adni.loni.usc.edu. We used tabulated values provided by the ADNI PET Core at the University of California Berkeley, where image quality control and preprocessing were performed. Here, we provide a brief description of image acquisition and of the processing performed by the ADNI PET Core. FTP PET scans were acquired over $30 \mathrm{~min}$ starting $75 \mathrm{~min}$ after an intravenous bolus injection of approximately $370 \mathrm{MBq}$ of radiotracer. Images were reconstructed to yield 6 time frames of 5 min each. FTP values were partial volume corrected using the geometric transfer matrix method (Rousset et al., 1998), and inferior cerebellar gray matter was used as the reference region to compute SUVRs. We computed a bilateral entorhinal cortex and a bilateral ITG volume-weighted average FTP SUVR using the tabular data provided in the ADNI database.

FDG PET scans were acquired over $30 \mathrm{~min}$ starting $30 \mathrm{~min}$ after an intravenous bolus injection. For FDG, we averaged the left and right temporal lobe components of the meta-ROI reported by (Landau et al., 2011), which falls largely within the ITG (but also includes part of the middle temporal gyrus).

FBP PET scans were acquired over 20 min and reconstructed to yield 4 time frames of 5 min each. FBP scans started $50 \mathrm{~min}$ after an intravenous bolus injection of approximately $370 \mathrm{MBq}$ of radiotracer. Whole cerebellum was used as the reference region to compute SUVR images. A global cortical amyloid index was calculated as the mean of frontal, lateral temporal, lateral parietal, and cingulate gray matter regions, and amyloid status was defined using an established cutoff of 1.11 (Schreiber et al., 2015).

\subsection{Statistical analyses}

We converted each continuous PET measure to $z$-scores using the mean and standard deviation at the visit corresponding to (or closest to) the baseline FTP PET scan for each participant. $z$-scores were computed separately for the BLSA and the ADNI. In the rest of the Methods section and in the Results, we refer to EC (or ITG) FTP SUVR $z$-score as EC (or ITG) tau, ITG PiB $R_{1} z$-score as ITG CBF, ITG FDG SUVR $z$-score as ITG glucose metabolism, 
and ITG ICV-adjusted volume $z$-score as ITG volume.

Statistical significance was defined as two-tailed $p<0.05$.

\subsubsection{Cross-sectional analysis}

Conditional independencies. The directed acyclic graph (DAG) motivating our cross-sectional analysis is presented in Figure1. The only conditional independence implied by this DAG is that EC tau and ITG neurodegeneration (i.e., CBF in the BLSA, glucose metabolism in the ADNI, and regional volume) are independent given amyloid, ITG tau, and the covariates (age, sex, $A P O E \varepsilon 4$ positivity, and education). We examined this conditional independency for each neurodegeneration measure using partial correlation. We also assessed more parsimonious versions of this model (i.e., DAGs with fewer edges) by testing their implied conditional independencies using partial correlations.

Cross-sectional associations. We investigated cross-sectional associations of amyloid with tau pathology in the EC and ITG using linear regression separately in the BLSA and ADNI. Models included age at FTP PET, sex, APOE $\varepsilon 4$ positivity, and years of education as covariates. We included EC tau as well as its interaction with amyloid as

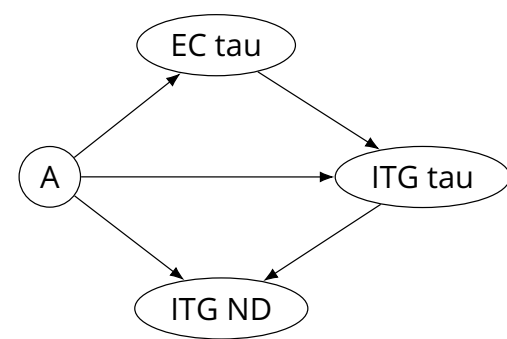

Figure 1. Directed acyclic graph representing the causal model for amyloid (A), tau, and neurodegeneration (ND). As ND measures, we investigated cerebral blood flow (in BLSA), glucose metabolism (in ADNI), and regional volume. Common covariates (age, sex, $A P O E \varepsilon 4$ positivity, and education) are not shown. explanatory variables in the model for ITG tau.

We then investigated the cross-sectional association of amyloid and ITG tau with ITG CBF (in the BLSA) or glucose metabolism (in the ADNI) using linear regression, adjusting for age at $\mathrm{PiB} P E T$, sex, $A P O E \varepsilon 4$ positivity, and years of education.

For each of these linear regression models in the BLSA, we investigated including race as an additional covariate. We did not investigate race as a covariate in the ADNI given its limited minority representation.

Causal mediation analysis. We combined these linear regression models in a causal mediation analysis to quantify (i) the effect of amyloid on ITG tau mediated by EC tau, and (ii) the effect of amyloid on ITG neurodegeneration (measured via $\mathrm{CBF}$ in the BLSA, glucose metabolism in ADNI, or regional volume) mediated by ITG tau. Finally, we assessed the sensitivity of our mediation analysis results to the sequential ignorability assumption that there are no unspecified confounders between the mediator and the outcome using a parametric sensitivity analysis based on the residual correlation between the mediator and the outcome (Imai) et al. 2010).

In the first analysis, the model for the outcome included an interaction between the exposure (amyloid) and the mediator (EC tau). Because of this, the causal mediation analysis involved the estimation of two mediation effects per model (one per amyloid group). These mediation effects are defined and interpreted within the counterfactual framework (Pearl, 2014). The average causal mediation effect (ACME) (also known as the natural indirect effect) for the $\mathrm{A}$ - group, denoted $\mathrm{ACME}_{\mathrm{A}-}$, is the expected difference in the outcome while amyloid status is fixed at A- and the mediator (tau) changes from the value attained or that would have been attained under amyloid negativity to the value under amyloid positivity for each individual. $\mathrm{ACME}_{\mathrm{A}+}$ is defined similarly, while fixing amyloid status at $\mathrm{A}+$ and changing the mediator from the value attained of would have been attained under $\mathrm{A}+$ to the value under $\mathrm{A}+$ for each individual. The average direct effect (ADE) (also known as the natural direct effect) for the $\mathrm{A}-$ group, denoted $\mathrm{ADE}_{\mathrm{A}_{-}}$, is the expected difference in the outcome as a result of changing amyloid status from $\mathrm{A}$ - to $\mathrm{A}+$ while keeping the mediator at the value observed or that would have been observed under $\mathrm{A}-$ for each individual. $\mathrm{ADE}_{\mathrm{A}+}$ is defined similarly, while keeping the mediator at the value observed or that would have been observed under A+ for each individual. For an A- individual, the value of the mediator had they been A+ is a counterfactual (and vice versa), since it involves imagining a scenario where the mediator is measured under an alternative amyloid status for that individual. ACME and ADE reflect the effect of amyloid on the outcome solely through tau (in the absence of the outcome's ability to respond to a change in amyloid status) and not through tau (in the absence of tau's ability to respond to a change in amyloid status), respectively. 


\subsubsection{Longitudinal analysis}

We investigated whether amyloid is associated with the rate of tau accumulation in the EC and ITG using linear mixed effects models. Outcome variables were EC and ITG tau. We included baseline age, time from baseline, sex, years of education, $A P O E \varepsilon 4$ positivity, amyloid status, and amyloid $\times$ time interaction as fixed effects. The model for ITG tau additionally included EC tau and its two-way interactions with amyloid and time as fixed effects.

We also investigated whether ITG tau is associated with the rate of change in ITG CBF, glucose metabolism, or volume. The outcome variable was ITG CBF (in the BLSA), glucose metabolism (in the ADNI), or volume. We included baseline age, time from baseline, sex, years of education, APOE $\varepsilon 4$ positivity, amyloid status, ITG tau, amyloid $\times$ ITG tau, amyloid $\times$ time, and ITG tau $\times$ time interactions as fixed effects.

All linear mixed effects models included a random intercept per participant. Random slopes were included only if the total number of observations was greater than the number of random effects to ensure parameter identifiability. The only model that met this criterion and included a random slope per participant was the model for ITG volume in the BLSA.

\subsection{Software}

We used https://causalfusion.net/ (Bareinboim and Pearl, 2016) to verify the logical expressions for the conditional independencies implied by our DAGs. All statistical analyses were conducted in R (https://cran r-project.org, version 4.0.3). We used the bnlearn (Scutari, 2010) package to test for conditional independence, lmerTest (Kuznetsova et al., 2017) to fit the linear mixed effects models, performance (Lüdecke et al., 2020) to check model diagnostics, mediation (Tingley et al., 2014) to perform the mediation analyses, tidyverse (Wickham et al. 2019) for data wrangling and plotting, ggExtra (Attali and Baker, 2019), ggthemes (Arnold, 2021), and wesanderson (Ram and Wickham, 2018) to generate plots, ggpubr (Kassambara, 2018) to create panel figures, stargazer (Hlavac, 2018) to tabulate model results, and knitr (Xie, 2018) to generate the manuscript directly incorporating results from $\mathrm{R}$.

\subsection{Code and data availability}

Code for performing statistical analyses and generating figures is provided in an open repository (https://gitlab.com/ bilgelm/tau_amyloid_neurodegeneration). Data from the BLSA are available upon request from the BLSA website (https://www.blsa.nih.gov). All requests are reviewed by the BLSA Data Sharing Proposal Review Committee. A deidentified version (with age bands instead of continuous age and excluding $A P O E$ genotype) of the cross-sectional BLSA data used in the statistical analyses is publicly available (https://doi.org/10.7910/DVN/YFJAZO). Data from the ADNI are available from the ADNI website (adni.loni.usc.edu).

\section{Results}

Participant characteristics are presented in Table 1. 103 BLSA and 122 ADNI participants were included in the analyses. A greater fraction of the participants were A+ in the ADNI compared to the BLSA (50\% vs. 31\%). Most of the BLSA participants were cognitively normal, whereas most of the ADNI participants had MCI. Regional FTP PET SUVRs were higher in the ADNI, but it is likely that these values are not directly comparable between the studies due to differences in scanners and image processing.

For the longitudinal analyses in the BLSA sample, there were a total of 167 FTP PET, 277 PiB PET, and 366 MRI scans. For BLSA participants with at least two scans, the median (interquartile range) time interval in years between last and first scan included in the longitudinal analyses was $2(1.6,2.2)$ for FTP PET, $3.9(2.4,4.8)$ for PiB PET, and $4.3(4,5.6)$ for MRI. In the ADNI sample, there were a total of 211 FTP PET, 194 FDG PET, and 229 MRI scans. For ADNI participants with at least two scans, the median (interquartile range) time interval in years between last and first scan included in the longitudinal analyses was $1.7(1.1,2.1)$ for FTP PET, $4.4(4,5)$ for FDG PET, and $1.3(1.1,2.1)$ for MRI. 
medRxiv preprint doi: https://doi.org/10.1101/2021.07.01.21259866; this version posted July 6, 2021. The copyright holder for this preprint (which was not certified by peer review) is the author/funder, who has granted medRxiv a license to display the preprint in perpetuity. This article is a US Government work. It is not subject to copyright under 17 USC 105 and is also made available for use under a CCO license.

Table 1. Participant characteristics. Continuous values are reported as median (interquartile range) and categorical values are reported as $N(\%)$. Age, diagnosis, PET measures, and MRI volumes are reported at the visit corresponding to or closest to the baseline FTP PET. A- = Amyloid negative, A+ = Amyloid positive, $\mathrm{ADNI}=$ Alzheimer's Disease Neuroimaging Initiative, $A P O E=$ Apolipoprotein $\mathrm{E}, \mathrm{BLSA}=$ Baltimore Longitudinal Study of Aging, FDG = fluorodeoxyglucose, FTP = flortaucipir, ICV-adj. = intracranial volume-adjusted, ITG $=$ inferior temporal gyrus, $\mathrm{MRI}=$ magnetic resonance imaging, $\mathrm{PiB}=$ Pittsburgh compound $\mathrm{B}, R_{1}=$ relative radiotracer delivery parameter, SUVR $=$ standardized update value ratio.

\begin{tabular}{|c|c|c|c|c|}
\hline & \multicolumn{2}{|c|}{ BLSA } & \multicolumn{2}{|c|}{ ADNI } \\
\hline & $\mathbf{A}-, \mathrm{N}=71$ & $\mathbf{A}+, \mathrm{N}=32$ & $\mathbf{A}-, \mathrm{N}=61$ & $\mathbf{A}+, \mathrm{N}=61$ \\
\hline Age at baseline FTP PET (years) & $77(70,83)$ & $78(73,87)$ & $76(70,81)$ & $75(71,80)$ \\
\hline \multicolumn{5}{|l|}{ Sex } \\
\hline Female & $43(61 \%)$ & $16(50 \%)$ & $21(34 \%)$ & $33(54 \%)$ \\
\hline Male & $28(39 \%)$ & $16(50 \%)$ & $40(66 \%)$ & $28(46 \%)$ \\
\hline \multicolumn{5}{|l|}{ Race } \\
\hline Asian or Pacific Islander & $4(6 \%)$ & $1(3 \%)$ & $1(2 \%)$ & - \\
\hline Black & $13(18 \%)$ & $4(12 \%)$ & $2(3 \%)$ & $4(7 \%)$ \\
\hline White & $54(76 \%)$ & $27(84 \%)$ & $57(93 \%)$ & $56(92 \%)$ \\
\hline Other & - & - & $1(2 \%)$ & $1(2 \%)$ \\
\hline \multicolumn{5}{|l|}{$A P O E$ status } \\
\hline$\varepsilon 4-$ & $51(72 \%)$ & $20(62 \%)$ & $52(85 \%)$ & $27(44 \%)$ \\
\hline$\varepsilon 4+$ & $20(28 \%)$ & $12(38 \%)$ & $9(15 \%)$ & $34(56 \%)$ \\
\hline Education (years) & $18(16,20)$ & $17(16,18)$ & $18(16,19)$ & $16(14,18)$ \\
\hline \multicolumn{5}{|l|}{ Diagnosis at baseline FTP PET } \\
\hline Cognitively normal & $70(99 \%)$ & $25(78 \%)$ & $11(18 \%)$ & $11(18 \%)$ \\
\hline Mild cognitive impairment & $1(1 \%)$ & $6(19 \%)$ & $50(82 \%)$ & $50(82 \%)$ \\
\hline Other impairment & - & $1(3 \%)$ & - & - \\
\hline \multicolumn{5}{|l|}{ PET measures at baseline FTP PET } \\
\hline Entorhinal cortex FTP SUVR & $1.02(0.93,1.1)$ & $1.07(1.01,1.23)$ & $1.91(1.74,2.34)$ & $2.44(2.02,2.97)$ \\
\hline ITG FTP SUVR & $1.24(1.19,1.32)$ & $1.37(1.29,1.49)$ & $1.92(1.8,2.02)$ & $2.05(1.91,2.62)$ \\
\hline ITG PiB $R_{1}$ & $0.88(0.86,0.9)$ & $0.85(0.82,0.9)$ & - & - \\
\hline ITG FDG SUVR & - & - & $1.22(1.17,1.31)$ & $1.21(1.12,1.32)$ \\
\hline ITG volume (ICV-adj. residual, $\mathrm{cm}^{3}$ ) & $-0.51(-1.45,0.45)$ & $-1.38(-2.54,0)$ & $-0.26(-1.67,1.1)$ & $-1.61(-3.12,0.41)$ \\
\hline \multicolumn{5}{|c|}{ No. FTP PET per participant } \\
\hline 1 & $41(58 \%)$ & $18(56 \%)$ & $34(56 \%)$ & $26(43 \%)$ \\
\hline 2 & $21(30 \%)$ & $9(28 \%)$ & $18(30 \%)$ & $22(36 \%)$ \\
\hline $3+$ & $9(13 \%)$ & $5(16 \%)$ & $9(15 \%)$ & $13(21 \%)$ \\
\hline \multicolumn{5}{|l|}{ No. PiB PET per participant } \\
\hline 1 & $16(23 \%)$ & $8(25 \%)$ & - & - \\
\hline 2 & $21(30 \%)$ & $7(22 \%)$ & - & - \\
\hline 3 & $18(25 \%)$ & $8(25 \%)$ & - & - \\
\hline $4+$ & $16(23 \%)$ & $9(28 \%)$ & - & - \\
\hline \multicolumn{5}{|l|}{ No. FDG PET per participant } \\
\hline 1 & - & - & $23(38 \%)$ & $37(61 \%)$ \\
\hline 2 & - & - & $32(52 \%)$ & $20(33 \%)$ \\
\hline 3 & - & - & $6(10 \%)$ & $4(7 \%)$ \\
\hline \multicolumn{5}{|l|}{ No. MRI per participant } \\
\hline 1 & $3(4 \%)$ & $1(3 \%)$ & $15(25 \%)$ & $27(44 \%)$ \\
\hline 2 & $12(17 \%)$ & $8(25 \%)$ & $29(48 \%)$ & $25(41 \%)$ \\
\hline 3 & $26(37 \%)$ & $11(34 \%)$ & $16(26 \%)$ & $9(15 \%)$ \\
\hline $4+$ & $30(42 \%)$ & $12(38 \%)$ & $1(2 \%)$ & - \\
\hline
\end{tabular}




\subsection{Cross-sectional analysis}

\subsubsection{Conditional independencies}

The partial correlation between EC tau and ITG CBF given amyloid status and all covariates was small and not statistically significant in the BLSA $(\rho=0.116, p=0.26)$. Similarly, the partial correlation between EC tau and ITG glucose metabolism was small and not statistically significant in the ADNI $(\rho=-0.169, p=0.07)$. The partial correlation between EC tau and ITG volume given amyloid status and all covariates was also small and not statistically significant in both the BLSA $(\rho=0.162, p=0.11)$ and the ADNI $(\rho=-0.166, p=0.075)$. These results are in agreement with the conditional independency implied by the DAG shown in Figure 1. None of the more parsimonious DAGs satisfied all implied conditional dependencies Appendix A.

\subsubsection{Cross-sectional associations}

Scatter plots of ITG vs. EC FTP SUVR (i.e., tau) are presented in Figure 2 Using linear regression models adjusted for age, sex, education, and $A P O E \varepsilon 4$ positivity, we found that EC tau was higher in the A+ compared to the $\mathrm{A}$ - group $(\beta=0.73, \mathrm{SE}=0.212, p<0.001$ in the $\mathrm{BLSA} ; \beta=0.443, \mathrm{SE}=0.191, p=0.023$ in the ADNI) (Table S1), and that higher EC tau was associated with higher ITG tau in both A- and A+ groups (in the A-group: $\beta=0.383, \mathrm{SE}=0.0968, p<0.001$ in the BLSA and $\beta=0.244, \mathrm{SE}=0.117, p=0.041$ in the ADNI; in the A+ group: $\beta=0.869, \mathrm{SE}=0.0812, p<0.001$ in the BLSA and $\beta=0.902, \mathrm{SE}=0.0728, p<0.001$ in the ADNI), with the A+ group exhibiting a stronger positive correlation (amyloid $\times \mathrm{EC}$ tau interaction term $\beta=0.486, \mathrm{SE}=0.126, p<0.001$ in the BLSA; $\beta=0.658, \mathrm{SE}=0.137, p<0.001$ in the ADNI) (Table S2).

\section{BLSA}

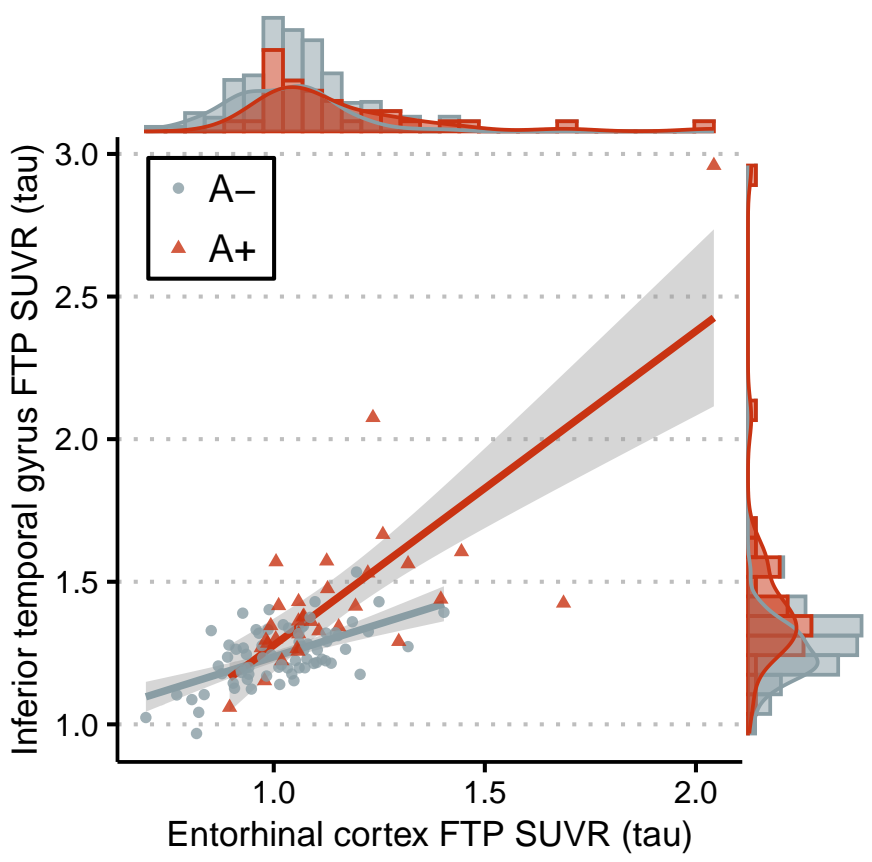

ADNI

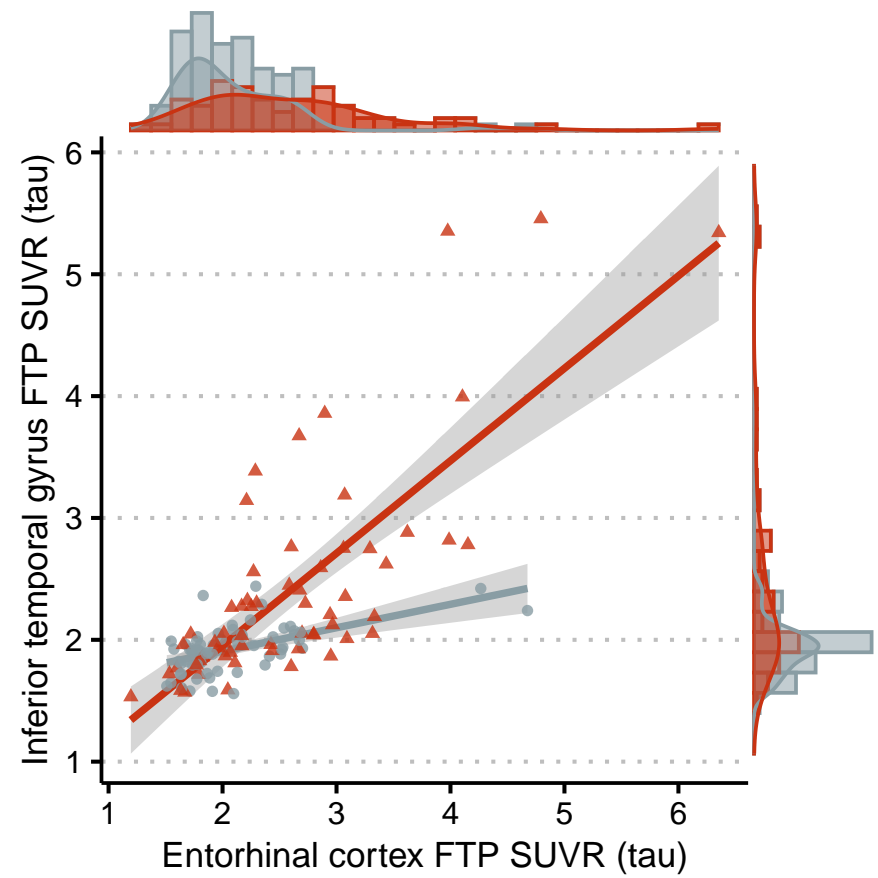

Figure 2. Scatter plots of inferior temporal gyrus vs. entorhinal cortex tau in the BLSA and ADNI. In linear regression models adjusted for age, sex, education, and $A P O E \varepsilon 4$ positivity, higher entorhinal tau was associated with higher inferior temporal gyrus tau in both amyloid-(A-) and A+ groups, with the A+ group exhibiting a stronger positive correlation. ADNI = Alzheimer's Disease Neuroimaging Initiative, BLSA = Baltimore Longitudinal Study of Aging, FTP = flortaucipir, SUVR = standardized uptake value ratio.

Scatter plots of ITG PiB $R_{1}$ (i.e., CBF) in the BLSA or FDG SUVR (i.e., glucose metabolism) in the ADNI vs. ITG FTP SUVR (i.e., tau) are presented in Figure 3 In linear regression models adjusted for age, sex, education, and 
$A P O E \varepsilon 4$ positivity, the inclusion of an amyloid $\times$ ITG tau interaction term did not yield a statistically significant difference in the residual sum of squares ( $F$-test $p=0.094$ in the BLSA and $p=0.22$ in the ADNI), and as a result, this interaction term was not included in the final models.

We did not find an association between amyloid group and ITG CBF $(\beta=-0.44, \mathrm{SE}=0.225, p=0.053)$ or glucose metabolism $(\beta=0.0923, \mathrm{SE}=0.204, p=0.653) z$-scores. A higher ITG tau was associated with a lower ITG CBF in the BLSA $(\beta=-0.31, \mathrm{SE}=0.104, p=0.004)$ and a lower ITG glucose metabolism in the ADNI $(\beta=-0.186, \mathrm{SE}=0.0926, p=0.047)$ (Table $\mathrm{S} 3$ ). When we included ITG volume as an additional covariate to adjust for partial volume effects, the association of ITG tau with ITG CBF in the BLSA remained statistically significant $(\beta=-0.28, \mathrm{SE}=0.102, p=0.008)$, but its association with ITG glucose metabolism in the ADNI was attenuated $(\beta=-0.04, \mathrm{SE}=0.093, p=0.669)$.

\section{BLSA}

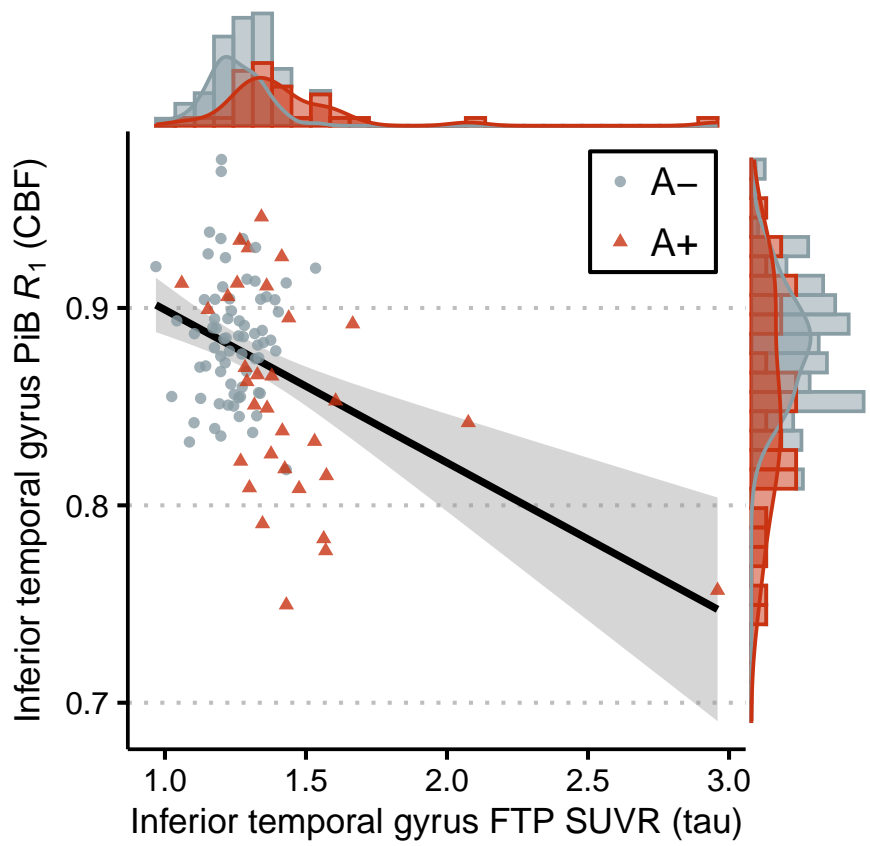

ADNI

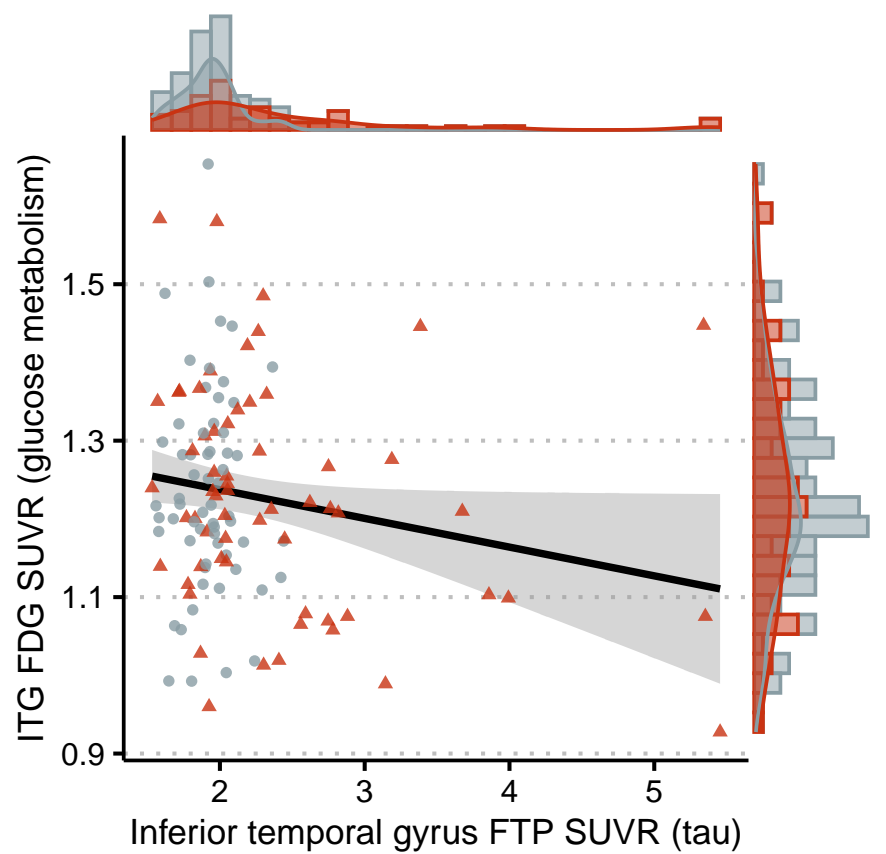

Figure 3. Scatter plots of cerebral blood flow (BLSA) or glucose metabolism (ADNI) in the inferior temporal gyrus vs. tau in the inferior temporal gyrus. In linear regression models adjusted for age, sex, education, and APOE $\varepsilon 4$ positivity, higher inferior temporal gyrus tau was associated with lower cerebral blood flow or glucose metabolism in the same region. ADNI = Alzheimer's Disease Neuroimaging Initiative, BLSA = Baltimore Longitudinal Study of Aging, FDG = fluorodeoxyglucose, $\mathrm{FTP}=$ flortaucipir, $\mathrm{PiB}=$ Pittsburgh compound $\mathrm{B}, R_{1}=$ relative delivery parameter, $\mathrm{SUVR}=$ standardized uptake value ratio.

Scatter plots of ITG volume (ICV-adjusted residuals) vs. ITG FTP SUVR (i.e., tau) are presented in Figure 4. In linear regression models adjusted for age, sex, education, and $A P O E \varepsilon 4$ positivity, the inclusion of an amyloid $\times$ ITG tau interaction term did not yield a statistically significant improvement in the residual sum of squares ( $F$-test $p=0.59$ in the BLSA and $p=0.19$ in the ADNI), and as a result, this interaction term was not included in the final models. We did not find an association between amyloid positivity and ITG volume either in the BLSA $(\beta=-0.118, \mathrm{SE}=0.238, p=0.621)$ or in the ADNI $(\beta=-0.267, \mathrm{SE}=0.191, p=0.165)$. A higher ITG tau was associated with a lower ITG volume in the ADNI $(\beta=-0.37, \mathrm{SE}=0.0866, p<0.001)$ but not in the BLSA $(\beta=-0.146, \mathrm{SE}=0.11, p=0.185)$ (Table S4).

\subsubsection{Causal mediation analysis}

Using causal mediation analysis, we found that the effect of amyloid on ITG tau was mediated by EC tau $\left(\mathrm{ACME}_{\mathrm{A}-}=\right.$ $0.28, p=0.0012, \mathrm{ACME}_{\mathrm{A}+}=0.634, p=0.0012$ in the BLSA; $\mathrm{ACME}_{\mathrm{A}-}=0.108, p=0.021, \mathrm{ACME}_{\mathrm{A}+}=0.4$, 


\section{BLSA}

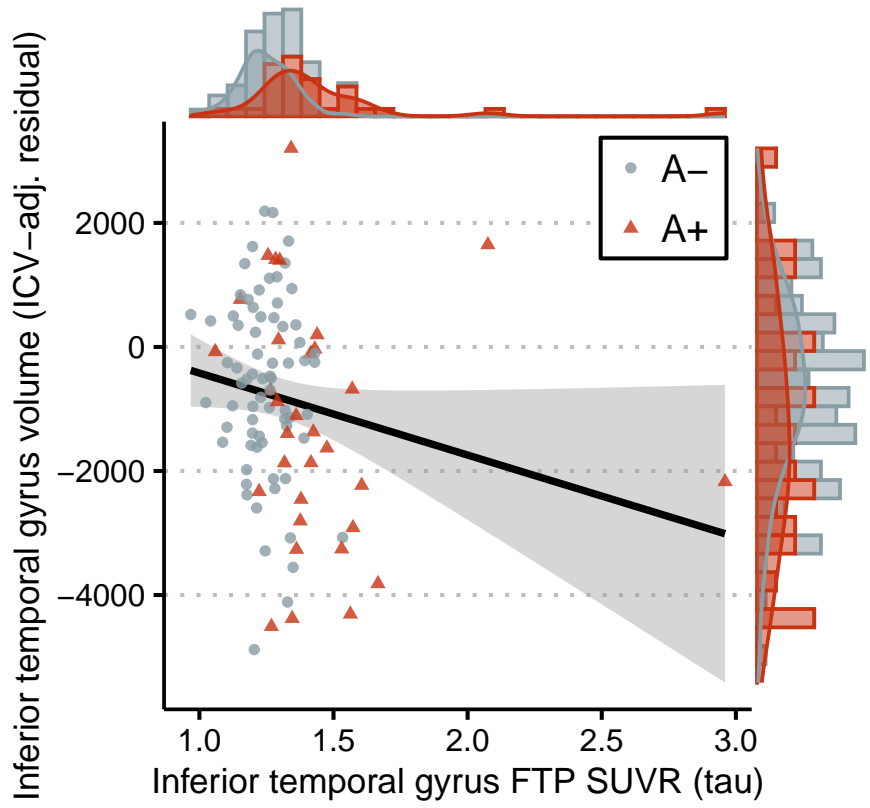

ADNI

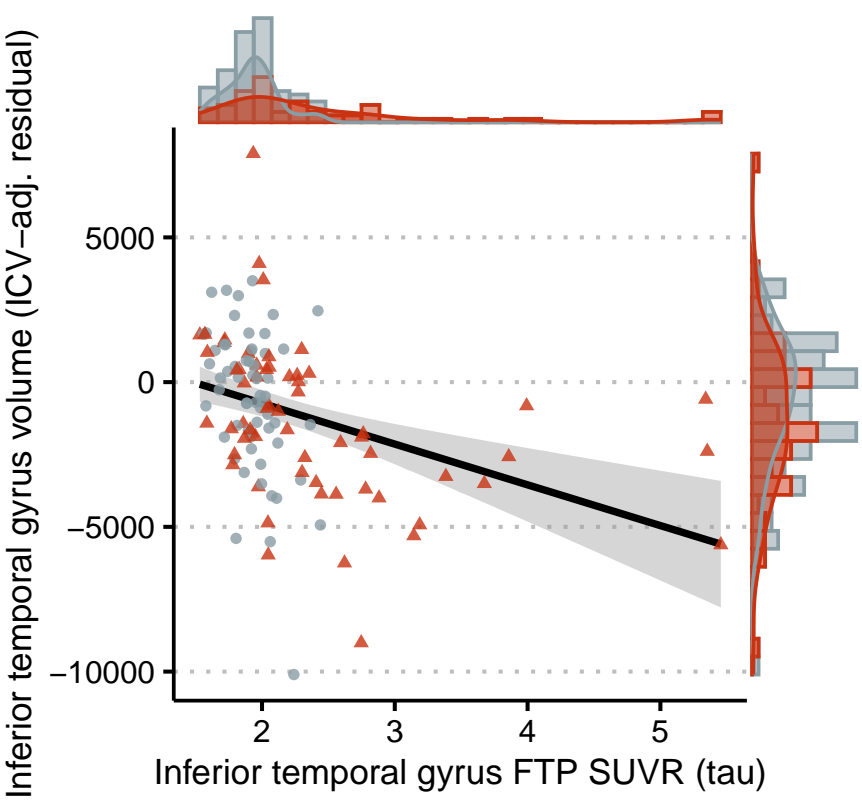

Figure 4. Scatter plots of inferior temporal gyrus volume (ICV-adjusted residuals) vs. tau in the BLSA and ADNI. In linear regression models adjusted for age, sex, education, and $A P O E \varepsilon 4$ positivity, higher inferior temporal gyrus tau was associated with lower volume in the ADNI, but not in the BLSA. ADNI = Alzheimer's Disease Neuroimaging Initiative, BLSA = Baltimore Longitudinal Study of Aging, FTP = flortaucipir, ICV = intracranial volume, SUVR = standardized uptake value ratio.

$p=0.021$ in the ADNI) (Figure 5p. The difference in ACME between the amyloid groups was not statistically significant in the BLSA $(p=0.29)$, but it was in the ADNI $(p=0.022)$. In both datasets, $\mathrm{ACME}_{\mathrm{A}+}$ was robust to violations of the assumption that there are no unspecified confounders between the mediator and the outcome (EC and ITG tau) (Figure S2).

We also found that the effect of amyloid on ITG CBF was mediated via ITG tau in the BLSA (ACME $=-0.292$, $p=0.014$ ), and that the effect of amyloid on ITG volume was mediated via ITG tau in the ADNI (ACME $=-0.234$, $p<0.001$ ) (Figure 6). Sensitivity analysis demonstrated that these ACME estimates are not as robust as our other findings to violations of the assumption of no unspecified confounders between the mediator (ITG tau) and the outcome (ITG CBF or volume) (S3 and S4, respectively). We do not report the results of the mediation analysis investigating ITG volume in the BLSA or ITG glucose metabolism in the ADNI since the associations between amyloid and these variables were small and not statistically significant (total effect on ITG volume in the BLSA $=-0.26, p=0.26$; total effect on ITG glucose metabolism in the ADNI $=-0.026, p=0.88$ ).

Including race as an additional covariate in the BLSA did not affect the statistical significance of these findings. We did not investigate race as a covariate in the ADNI sample given its limited minority representation.

\subsection{Longitudinal analysis}

In the BLSA, we did not find a statistically significant association between amyloid and rate of change in EC tau $(\beta=0.0789, \mathrm{SE}=0.0605, p=0.198)$, or between amyloid or baseline $\mathrm{EC}$ tau and rate of change in ITG tau $(\beta=-0.0297, \mathrm{SE}=0.0273, p=0.281)$. In the ADNI, A+ individuals had steeper increases in EC tau over time $(\beta=0.0675, \mathrm{SE}=0.0328, p=0.043)$ (Table S5). A higher baseline EC tau was associated with a steeper increase in ITG tau over time $(\beta=0.0799, \mathrm{SE}=0.0199, p<0.001)$. The inclusion of an amyloid $\times \mathrm{EC}$ tau $\times$ time interaction term in the model for ITG tau did not yield a statistically significant difference in the log-likelihood $\left(\chi^{2}\right.$-test $\left.p=0.053\right)$, and as a result, this interaction term was not included in the final model.

We did not find an association between amyloid status and the rate of change in ITG CBF $(\beta=0.0145, \mathrm{SE}=$ 

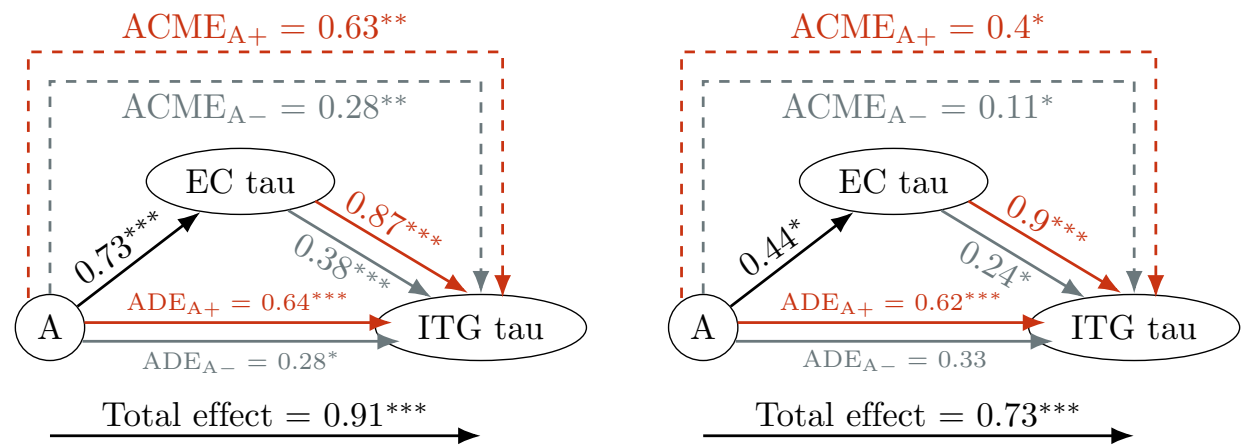

Figure 5. Causal mediation analysis results for the model investigating the relationships among amyloid group (A) and tau in the entorhinal cortex (EC) and the inferior temporal gyrus (ITG) in the BLSA (left) and the ADNI (right). The effect of amyloid on ITG tau was mediated by EC tau. ACME = average causal mediation effect, ADE $=$ average direct effect. ${ }^{*} p<0.05 ;{ }^{* *} p<0.01 ; * * * p<0.001$.
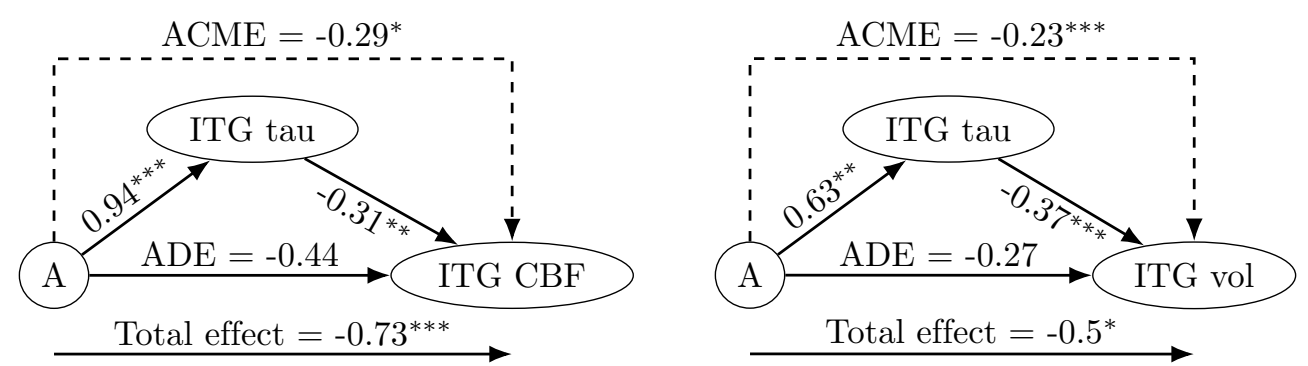

Figure 6. Causal mediation analysis results for the model investigating the relationships among amyloid group (A), tau in the inferior temporal gyrus (ITG), and neurodegeneration. The neurodegeneration measure shown is cerebral blood flow (CBF) in the ITG in the BLSA (left), and ITG volume in the ADNI (right). The effect of amyloid on ITG CBF (in BLSA) and volume (in ADNI) was mediated via ITG tau, and its direct effect was not statistically significant. The effect of amyloid on ITG volume was mediated via ITG tau. ACME = average causal mediation effect, $\mathrm{ADE}=$ average direct effect, ADNI = Alzheimer's Disease Neuroimaging Initiative, BLSA = Baltimore Longitudinal Study of Aging. ${ }^{*} p<0.05 ;{ }^{* *} p<0.01 ;{ }^{* * *} p<0.001$.

$0.0313, p=0.644$ in the BLSA $)$, glucose metabolism $(\beta=-0.00672, \mathrm{SE}=0.0399, p=0.867$ in the ADNI), or volume $(\beta=-0.0129, \mathrm{SE}=0.0228, p=0.573$ in the $\mathrm{BLSA} ; \beta=-0.00217, \mathrm{SE}=0.0407, p=0.958$ in the ADNI). A higher baseline ITG tau was associated with a steeper decrease in ITG glucose metabolism $(\beta=-0.0901, \mathrm{SE}=$ $0.0396, p=0.026)$ (Table S7) and ITG volume over time $(\beta=-0.0487, \mathrm{SE}=0.0139, p<0.001$ in the BLSA; $\beta=-0.0644, \mathrm{SE}=0.0237, p=0.008$ in the $\mathrm{ADNI}$ ) (Table $\mathrm{S} 8$ ), but not with the rate of change in ITG CBF $(\beta=0.029, \mathrm{SE}=0.019, p=0.13)$.

\section{Discussion}

In both the BLSA and the ADNI, greater EC tau was associated with greater ITG tau, and this association was stronger among amyloid positive individuals. These findings suggest that amyloid facilitates the spread of tau from the MTL to the neocortex. Amyloid's association with ITG tau was in part mediated by EC tau: EC tau mediated $50 \%$ of the total effect in the BLSA and 35\% in the ADNI. Higher ITG tau was associated with several proxies of neurodegeneration in the same region, including CBF, glucose metabolism, and volume (in the ADNI only). While we did not find a direct effect of amyloid positivity on neurodegeneration, it had an indirect effect via tau pathology on CBF (BLSA) and volume (ADNI). The indirect effect of amyloid via ITG tau accounted for $40 \%$ of the total effect on ITG CBF, and $47 \%$ of the total effect on ITG volume in the ADNI, but the statistical significance of these indirect effect estimates was sensitive to the assumption that there were no unspecified confounders of ITG tau and neurodegeneration in our model. 
In longitudinal analyses, we found that amyloid positivity is associated with steeper increases in EC tau, and that greater EC tau at baseline is associated with steeper increases in ITG tau in the ADNI. Greater ITG tau at baseline was associated with steeper decreases in glucose metabolism in the same region. The finding that greater ITG tau at baseline is associated with faster volume loss was observed in both the BLSA and the ADNI.

Our findings have therapeutic implications. The causal mediation results suggest that treating EC tau alone, ceteris paribus, would likely be insufficient in preventing the neocortical spread of tau, since the direct path between amyloid and ITG tau would not be affected. Similarly, preventing amyloid alone among individuals who already have some level of EC tau would also likely be insufficient, since EC tau would continue to spread to the neocortex. However, given the synergistic association between amyloid and EC tau in neocortical propagation, targeting either pathology would be expected to slow down the spread of tau to the neocortex. A recent clinical trial of donanemab, an anti-amyloid monoclonal antibody, conducted among patients with early symptomatic AD failed to find a difference in global tau load between treatment and placebo groups, but there was a greater reduction in tau in frontal and temporal regions in the treated group (Mintun et al., 2021). An amyloid intervention earlier in the disease course, combined with a tau-targeting therapy, might yield more pronounced effects on neocortical tau. Considering the recent FDA approval of aducanumab, another anti-amyloid monoclonal antibody, it is vital that the potential mechanism of action of anti-amyloid drugs for reducing or preventing neurodegeneration be elucidated as soon as possible. Such information will inform future directions for anti-amyloid and anti-tau accumulation strategies.

Tau, rather than amyloid, might be the main driver of neurodegeneration. In the recently completed phase 2 trial among patients with mild $\mathrm{AD}$ of AADvac1, an active immunotherapy against pathological tau, neurodegeneration was slower in the treatment group, as evidenced by an attenuated increase in neurofilament light (Novak et al., 2021). This clinical trial result supports our model assumption that tau is a contributor to neurodegeneration. However, our causal mediation results also suggest that tau pathology does not explain the entire degree of neurodegeneration, in alignment with previous reports (Gómez-Isla et al. 1997). We did not observe an interaction between amyloid and neocortical tau in relation to neurodegeneration, while such a synergistic relationship has been reported in transgenic mice (Pooler et al. 2015) and human studies (Hanseeuw et al., 2017). The lack of such a relationship in our study might be due to small sample sizes or the exclusion of participants with dementia.

The cross-sectional finding that tau pathology was associated with volume in the ADNI but not in the BLSA might be explained by differences in the composition of the samples: most of the ADNI participants included in our analyses had MCI, whereas most BLSA participants were cognitively normal. This difference between the two samples suggests that the detrimental effects of tau pathology on volume loss might not be evident until later stages of disease. Indeed, we found that higher tau at baseline was associated with steeper prospective volume declines in both the BLSA and ADNI. Supporting this hypothesis, a simulation study based on quantitative neuron loss and neurofibrillary tangle (NFT) formation data suggested that neurons harboring NFTs may survive for about two decades (Morsch et al., 1999). Our findings in the BLSA might reflect differences in the temporal sequence of changes. CBF might exhibit neuropathology-associated changes while individuals are cognitively normal, prior to declines in regional brain volumes (Sperling et al., 2011). This could explain the absence of a cross-sectional association of tau with volume in the BLSA and the presence of an association with CBF.

Our longitudinal findings in the ADNI provide support for most of the causal directions assumed in our DAG (Fig. 1), with one notable exception being the path from amyloid to neurodegeneration. In analyses adjusted for tau, we did not find an association between amyloid positivity and rate of change in any of the neurodegeneration measures we assessed. The lack of an association suggests that the direct effect of fibrillar amyloid on neurodegenerative change is small and requires larger samples to be detected, or that fibrillar amyloid is not a direct player in CBF, glucose metabolism, or volume loss changes among individuals without dementia.

Limitations of our study include small samples and lack of measures for other factors implicated in tau spread (i.e., neuroinflammation or synaptic markers), which prevents the analysis of competing mechanisms. We used binary amyloid status based on fibrillar amyloid burden, but a measure of soluble amyloid could have been more appropriate given the increasing evidence that soluble forms induce tau hyperphosphorylation and may be more closely involved in neurodegeneration rather than fibrillar amyloid. However, concentrations of soluble $\mathrm{A} \beta$ oligomers correlate with the amount of $\mathrm{A} \beta$ plaques, albeit weakly, among individuals without dementia (Esparza et al., 2013). Our causal model is likely an incomplete and simplified version of reality. While we assumed a unidirectional path from amyloid to tau, it is possible that these pathologies exist within a feedback loop. One study suggested that amyloid and tau pathology reinforce each other (Pooler et al. 2015). There may be mechanisms by which cortical fibrillar amyloid deposition can influence MTL tau pathology; for example, amyloid pathology is associated with axonal dystrophy (McKee et al. 
1991), which might be a mechanism by which neocortical amyloid exerts retrograde effects. Furthermore, there are differing schools of thought about the sequence of changes in CBF and tau (Korte et al., 2020), but it is unclear whether PET measures of CBF would reliably detect the types of early changes that are hypothesized to lead to tau pathology. Extensive longitudinal data will be necessary to determine the directions of these associations.

An important strength of our study is the validation of several of our findings in two longitudinal cohorts. In particular, the cross-sectional causal mediation analysis findings regarding amyloid-facilitated propagation of tau from the MTL to the neocortex were highly consistent in the BLSA and the ADNI. Based on this consistency, we expect these findings to be generalizable to similar samples of individuals without dementia. We employed sensitivity analysis to assess an important assumption of causal mediation analysis, namely, that the assignment of the mediator values (EC or ITG tau, depending on the model) is random when amyloid status and observed covariates are adjusted for, and demonstrated that our findings regarding tau spread from the MTL to the neocortex are relatively robust to this assumption.

In conclusion, our study confirms previous findings that amyloid is associated with greater tau pathology in both the MTL and the neocortex, and provides further evidence for the facilitating role of amyloid in the neocortical propagation of tau among cognitively normal individuals and in MCI. Since entorhinal tau mediated only up to half of the association of amyloid with neocortical tau, we speculate that interventions to reduce only MTL tau or only amyloid among individuals who already have MTL tau might slow but not fully prevent the neocortical spread of tau. Preventing neocortical tau early in the course of neuropathology might be an important element in preventing cortical neurodegeneration, including lower CBF, hypometabolism, and volume loss. Combination treatments that impact both amyloid and tau early in the course of disease may be necessary to prevent or slow progression.

\section{Acknowledgments}

We thank Dr. Andrea Shafer and Dr. Firat Bilgel for their thoughtful feedback. We are grateful to the BLSA participants and staff for their dedication to these studies. We thank the Johns Hopkins PET facility staff, the NIA 3T MRI facility staff, Alisa Bannerjee and Wendy Elkins for their assistance with coordination and data collection for the BLSA PET studies, the Center for Biomedical Image Computing and Analytics for providing MUSE labels and their contributions to MRI analysis, and Avid Radiopharmaceuticals for enabling the use of the ${ }^{18} \mathrm{~F}$-flortaucipir tracer and proving the precursor. Avid Radiopharmaceuticals did not provide direct funding for any of the PET studies nor personnel and were not involved in data analysis or interpretation. This study was supported by the Intramural Research Program of the National Institute on Aging, NIH.

Data collection and sharing for this project was funded by the Alzheimer's Disease Neuroimaging Initiative (ADNI) (National Institutes of Health Grant U01 AG024904) and DOD ADNI (Department of Defense award number W81XWH-12-2-0012). ADNI is funded by the National Institute on Aging, the National Institute of Biomedical Imaging and Bioengineering, and through generous contributions from the following: AbbVie, Alzheimer's Association; Alzheimer's Drug Discovery Foundation; Araclon Biotech; BioClinica, Inc.; Biogen; Bristol-Myers Squibb Company; CereSpir, Inc.; Cogstate; Eisai Inc.; Elan Pharmaceuticals, Inc.; Eli Lilly and Company; EuroImmun; F. Hoffmann-La Roche Ltd and its affiliated company Genentech, Inc.; Fujirebio; GE Healthcare; IXICO Ltd.; Janssen Alzheimer Immunotherapy Research \& Development, LLC.; Johnson \& Johnson Pharmaceutical Research \& Development LLC.; Lumosity; Lundbeck; Merck \& Co., Inc.; Meso Scale Diagnostics, LLC.; NeuroRx Research; Neurotrack Technologies; Novartis Pharmaceuticals Corporation; Pfizer Inc.; Piramal Imaging; Servier; Takeda Pharmaceutical Company; and Transition Therapeutics. The Canadian Institutes of Health Research is providing funds to support ADNI clinical sites in Canada. Private sector contributions are facilitated by the Foundation for the National Institutes of Health (www.fnih.org). The grantee organization is the Northern California Institute for Research and Education, and the study is coordinated by the Alzheimer's Therapeutic Research Institute at the University of Southern California. ADNI data are disseminated by the Laboratory for Neuro Imaging at the University of Southern California.

\section{References}

Albrecht, D., A. Lisette Isenberg, J. Stradford, T. Monreal, A. Sagare, M. Pachicano, M. Sweeney, A. Toga, B. Zlokovic, H. Chui, E. Joe, L. Schneider, P. Conti, K. Jann, and J. Pa 2020. Associations between vascular function and Tau PET are associated with global cognition and amyloid. Journal of Neuroscience, 40(44):8573-8586. 
Arnold, J. B. 2021. ggthemes: Extra Themes, Scales and Geoms for 'ggplot2'.

Attali, D. and C. Baker 2019. ggExtra: Add Marginal Histograms to 'ggplot2', and More 'ggplot2' Enhancements.

Bareinboim, E. and J. Pearl 2016. Causal inference and the data-fusion problem. Proceedings of the National Academy of Sciences of the United States of America, 113(27):7345-7352.

Berron, D., J. W. Vogel, P. S. Insel, J. B. Pereira, L. Xie, L. E. M. Wisse, P. A. Yushkevich, S. Palmqvist, N. MattssonCarlgren, E. Stomrud, R. Smith, O. Strandberg, and O. Hansson 2021. Early stages of tau pathology and its associations with functional connectivity, atrophy and memory. Brain, (2021).

Bilgel, M., L. Beason-Held, Y. An, Y. Zhou, D. F. Wong, and S. M. Resnick 2019. Longitudinal evaluation of surrogates of regional cerebral blood flow computed from dynamic amyloid PET imaging. Journal of Cerebral Blood Flow $\&$ Metabolism.

Bischof, G. N., F. Jessen, K. Fliessbach, J. Dronse, J. Hammes, B. Neumaier, O. Onur, G. R. Fink, J. Kukolja, A. Drzezga, and T. van Eimeren 2016. Impact of tau and amyloid burden on glucose metabolism in Alzheimer's disease. Annals of Clinical and Translational Neurology, 3(12):934-939.

Buckley, R. F., E. C. Mormino, J. Chhatwal, A. P. Schultz, J. S. Rabin, D. M. Rentz, D. Acar, M. J. Properzi, J. Dumurgier, H. Jacobs, T. Gomez-Isla, K. A. Johnson, R. A. Sperling, and B. J. Hanseeuw 2019. Associations between baseline amyloid, sex, and APOE on subsequent tau accumulation in cerebrospinal fluid. Neurobiology of Aging, 78:178-185.

Calafate, S., A. Buist, K. Miskiewicz, V. Vijayan, G. Daneels, B. de Strooper, J. de Wit, P. Verstreken, and D. Moechars 2015. Synaptic Contacts Enhance Cell-to-Cell Tau Pathology Propagation. Cell Reports, 11(8):1176-1183.

Carbonell, F., A. P. Zijdenbos, and B. J. Bedell 2020. Spatially Distributed Amyloid- $\beta$ Reduces Glucose Metabolism in Mild Cognitive Impairment. Journal of Alzheimer's Disease, 73(2):543-557.

Chételat, G., V. L. Villemagne, P. Bourgeat, K. E. Pike, G. Jones, D. Ames, K. a. Ellis, C. Szoeke, R. N. Martins, G. J. O'Keefe, O. Salvado, C. L. Masters, C. C. Rowe, and Australian Imaging Biomarkers and Lifestyle Research Group 2010. Relationship between atrophy and $\beta$-amyloid deposition in Alzheimer disease. Annals of Neurology, 67(3):317-324.

De Calignon, A., M. Polydoro, M. Suárez-Calvet, C. William, D. H. Adamowicz, K. J. Kopeikina, R. Pitstick, N. Sahara, K. H. Ashe, G. A. Carlson, T. L. Spires-Jones, and B. T. Hyman 2012. Propagation of Tau Pathology in a Model of Early Alzheimer's Disease. Neuron, 73(4):685-697.

Desikan, R. S., F. Ségonne, B. Fischl, B. T. Quinn, B. C. Dickerson, D. Blacker, R. L. Buckner, A. M. Dale, R. P. Maguire, B. T. Hyman, M. S. Albert, and R. J. Killiany 2006. An automated labeling system for subdividing the human cerebral cortex on MRI scans into gyral based regions of interest. NeuroImage, 31(3):968-980.

Doshi, J., G. Erus, M. Habes, and C. Davatzikos 2019. DeepMRSeg: A convolutional deep neural network for anatomy and abnormality segmentation on MR images. arXiv.

Doshi, J., G. Erus, Y. Ou, S. M. Resnick, R. C. Gur, R. E. Gur, T. D. Satterthwaite, S. Furth, and C. Davatzikos 2016. MUSE: MUlti-atlas region Segmentation utilizing Ensembles of registration algorithms and parameters, and locally optimal atlas selection. NeuroImage, 127(2016):186-195.

Esparza, T. J., H. Zhao, J. R. Cirrito, N. J. Cairns, R. J. Bateman, D. M. Holtzman, and D. L. Brody 2013. Amyloidbeta oligomerization in Alzheimer dementia versus high-pathology controls. Annals of Neurology, 73(1):104-119.

Fuld, P. A. 1978. Psychological testing in the differential diagnosis of the dementias. In Alzheimer's disease: Senile dementia and related disorders, R. Katzman, R. D. Terry, and K. L. Bick, eds., Pp. 185-193. New York, NY: Raven Press. 
medRxiv preprint doi: https://doi.org/10.1101/2021.07.01.21259866; this version posted July 6, 2021. The copyright holder for this preprint

(which was not certified by peer review) is the author/funder, who has granted medRxiv a license to display the preprint in perpetuity. This article is a US Government work. It is not subject to copyright under 17 USC 105 and is also made available for use under a CCO license.

Gómez-Isla, T., R. Hollister, H. West, S. Mui, J. H. Growdon, R. C. Petersen, J. E. Parisi, and B. T. Hyman 1997. Neuronal loss correlates with but exceeds neurofibrillary tangles in Alzheimer's disease. Annals of Neurology, 41(1):17-24.

Hanseeuw, B. J., R. A. Betensky, A. P. Schultz, K. V. Papp, E. C. Mormino, J. Sepulcre, J. S. Bark, D. M. Cosio, M. LaPoint, J. P. Chhatwal, D. M. Rentz, R. A. Sperling, and K. A. Johnson 2017. Fluorodeoxyglucose metabolism associated with tau-amyloid interaction predicts memory decline. Annals of Neurology, 81(4):583-596.

Harrison, T. M., R. La Joie, A. Maass, S. L. Baker, K. Swinnerton, L. Fenton, T. J. Mellinger, L. Edwards, J. Pham, B. L. Miller, G. D. Rabinovici, and W. J. Jagust 2018. Longitudinal tau accumulation and atrophy in aging and Alzheimer's disease. Annals of Neurology.

Hlavac, M. 2018. stargazer: Well-formatted regression and summary statistics tables.

Iaccarino, L., G. Tammewar, N. Ayakta, S. L. Baker, A. Bejanin, A. L. Boxer, M. L. Gorno-Tempini, M. Janabi, J. H. Kramer, A. Lazaris, S. N. Lockhart, B. L. Miller, Z. A. Miller, J. P. O’Neil, R. Ossenkoppele, H. J. Rosen, D. R. Schonhaut, W. J. Jagust, and G. D. Rabinovici 2018. Local and distant relationships between amyloid, tau and neurodegeneration in Alzheimer's Disease. NeuroImage: Clinical, 17(September 2017):452-464.

Imai, K., L. Keele, and D. Tingley 2010. A General Approach to Causal Mediation Analysis. Psychological Methods, 15(4):309-334.

Jack, C. R., K. Twomey, A. R. Zinsmeister, F. W. Sharbrough, C. Petersen, and G. D. Cascino 1989. Anterior temporal lobes and hippocampal formations: normative volumetric measurements from MR images in young adults. Radiology, 172:549-554.

Jack, C. R., H. J. Wiste, C. G. Schwarz, V. J. Lowe, M. L. Senjem, P. Vemuri, S. D. Weigand, T. M. Therneau, D. S. Knopman, J. L. Gunter, D. T. Jones, J. Graff-Radford, K. Kantarci, R. O. Roberts, M. M. Mielke, M. M. Machulda, and R. C. Petersen 2018. Longitudinal tau PET in ageing and Alzheimer's disease. Brain, 141(5):1517-1528.

Jack, C. R., H. J. Wiste, S. D. Weigand, T. M. Therneau, D. S. Knopman, V. Lowe, P. Vemuri, M. M. Mielke, R. O. Roberts, M. M. Machulda, M. L. Senjem, J. L. Gunter, W. A. Rocca, and R. C. Petersen 2017. Age-specific and sex-specific prevalence of cerebral $\beta$-amyloidosis, tauopathy, and neurodegeneration in cognitively unimpaired individuals aged 50-95 years: a cross-sectional study. The Lancet Neurology, 4422(17).

Jin, M., N. Shepardson, T. Yang, G. Chen, D. Walsh, and D. J. Selkoe 2011. Soluble amyloid $\beta$-protein dimers isolated from Alzheimer cortex directly induce Tau hyperphosphorylation and neuritic degeneration. Proceedings of the National Academy of Sciences of the United States of America, 108(14):5819-5824.

Kassambara, A. 2018. ggpubr: 'ggplot2' based publication ready plots.

Korte, N., R. Nortley, and D. Attwell 2020. Cerebral blood flow decrease as an early pathological mechanism in Alzheimer's disease. Acta Neuropathologica, 140(6):793-810.

Kuznetsova, A., P. B. Brockhoff, and R. H. B. Christensen 2017. lmerTest Package: Tests in Linear Mixed Effects Models. Journal of Statistical Software, 82(13).

La Joie, R., A. V. Visani, S. L. Baker, J. A. Brown, V. Bourakova, J. Cha, K. Chaudhary, L. Edwards, L. Iaccarino, M. Janabi, O. H. Lesman-Segev, Z. A. Miller, D. C. Perry, J. P. O’Neil, J. Pham, J. C. Rojas, H. J. Rosen, W. W. Seeley, R. M. Tsai, B. L. Miller, W. J. Jagust, and G. D. Rabinovici 2020. Prospective longitudinal atrophy in Alzheimer's disease correlates with the intensity and topography of baseline tau-PET. Science Translational Medicine, 12(524):eaau5732.

Landau, S. M., D. Harvey, C. M. Madison, R. A. Koeppe, E. M. Reiman, N. L. Foster, M. W. Weiner, and W. J. Jagust 2011. Associations between cognitive, functional, and FDG-PET measures of decline in AD and MCI. Neurobiology of Aging, 32(7):1207-1218.

Liu, L., V. Drouet, J. W. Wu, M. P. Witter, S. A. Small, C. Clelland, and K. Duff 2012. Trans-synaptic spread of tau pathology in vivo. PLoS ONE, 7(2):1-9. 
medRxiv preprint doi: https://doi.org/10.1101/2021.07.01.21259866; this version posted July 6, 2021. The copyright holder for this preprint

(which was not certified by peer review) is the author/funder, who has granted medRxiv a license to display the preprint in perpetuity.

This article is a US Government work. It is not subject to copyright under 17 USC 105 and is also made available for use under a CCO license.

Lüdecke, D., D. Makowski, P. Waggoner, and I. Patil 2020. performance: Assessment of Regression Models Performance.

McKee, A. C., K. S. Kosik, and N. W. Kowall 1991. Neuritic pathology and dementia in alzheimer's disease. Annals of Neurology, 30(2):156-165.

Mintun, M. A., A. C. Lo, C. Duggan Evans, A. M. Wessels, P. A. Ardayfio, S. W. Andersen, S. Shcherbinin, J. Sparks, J. R. Sims, M. Brys, L. G. Apostolova, S. P. Salloway, and D. M. Skovronsky 2021. Donanemab in Early Alzheimer's Disease. New England Journal of Medicine, 384(18):1691-1704.

Morris, J. C. 1993. The Clinical Dementia Rating (CDR): Current version and scoring rules. Neurology, 43(11):24122414.

Morsch, R., W. Simon, and P. D. Coleman 1999. Neurons may live for decades with neurofibrillary tangles. Journal of Neuropathology \&3 Experimental Neurology, 58(2):188-197.

Nelson, P. T., I. Alafuzoff, E. H. Bigio, C. Bouras, H. Braak, N. J. Cairns, R. J. Castellani, B. J. Crain, P. Davies, K. Del Tredici, C. Duyckaerts, M. P. Frosch, V. Haroutunian, P. R. Hof, C. M. Hulette, B. T. Hyman, T. Iwatsubo, K. A. Jellinger, G. A. Jicha, E. Kövari, W. A. Kukull, J. B. Leverenz, S. Love, I. R. Mackenzie, D. M. Mann, E. Masliah, A. C. McKee, T. J. Montine, J. C. Morris, J. A. Schneider, J. A. Sonnen, D. R. Thal, J. Q. Trojanowski, J. C. Troncoso, T. Wisniewski, R. L. Woltjer, and T. G. Beach 2012. Correlation of Alzheimer disease neuropathologic changes with cognitive status: a review of the literature. Journal of Neuropathology and Experimental Neurology, $71(5): 362-381$.

Novak, P., B. Kovacech, S. Katina, R. Schmidt, P. Scheltens, E. Kontsekova, S. Ropele, L. Fialova, M. Kramberger, N. Paulenka-Ivanovova, M. Smisek, J. Hanes, E. Stevens, A. Kovac, S. Sutovsky, V. Parrak, P. Koson, M. Prcina, J. Galba, M. Cente, T. Hromadka, P. Filipcik, J. Piestansky, M. Samcova, C. Prenn-Gologranc, R. Sivak, L. Froelich, M. Fresser, M. Rakusa, J. Harrison, J. Hort, M. Otto, D. Tosun, M. Ondrus, B. Winblad, M. Novak, and N. Zilka 2021. ADAMANT: a placebo-controlled randomized phase 2 study of AADvac1, an active immunotherapy against pathological tau in Alzheimer's disease. Nature Aging, 1(6):521-534.

Ossenkoppele, R., D. R. Schonhaut, M. Schöll, S. N. Lockhart, N. Ayakta, S. L. Baker, J. P. O’Neil, M. Janabi, A. Lazaris, A. Cantwell, J. Vogel, M. Santos, Z. A. Miller, B. M. Bettcher, K. A. Vossel, J. H. Kramer, M. L. Gorno-Tempini, B. L. Miller, W. J. Jagust, and G. D. Rabinovici 2016. Tau PET patterns mirror clinical and neuroanatomical variability in Alzheimer's disease. Brain, 139(5):1551-1567.

Pearl, J. 2014. Interpretation and identification of causal mediation. Psychological Methods, 19(4):459-481.

Petersen, R. C. 2004. Mild cognitive impairment as a diagnostic entity. Journal of Internal Medicine, 256(3):183-194.

Petersen, R. C., P. S. Aisen, L. A. Beckett, M. C. Donohue, A. C. Gamst, D. J. Harvey, C. R. Jack, W. J. Jagust, L. M. Shaw, and A. W. Toga 2010. Alzheimer's Disease Neuroimaging Initiative (ADNI) clinical characterization. Neurology, 74(3):201-209.

Petersen, R. C., H. J. Wiste, S. D. Weigand, W. A. Rocca, R. O. Roberts, M. M. Mielke, V. J. Lowe, D. S. Knopman, V. S. Pankratz, M. M. Machulda, Y. E. Geda, and C. R. Jack Jr. 2016. Association of elevated amyloid levels with cognition and biomarkers in cognitively normal people from the community. JAMA Neurology, 73(1):85-92.

Pooler, A. M., M. Polydoro, E. A. Maury, S. B. Nicholls, S. M. Reddy, S. Wegmann, C. William, L. Saqran, O. CagsalGetkin, R. Pitstick, D. R. Beier, G. A. Carlson, T. L. Spires-Jones, and B. T. Hyman 2015. Amyloid accelerates tau propagation and toxicity in a model of early Alzheimer's disease. Acta Neuropathologica Communications, 3(1):14.

Ram, K. and H. Wickham 2018. wesanderson: A Wes Anderson Palette Generator.

Rousset, O. G., Y. Ma, and A. C. Evans 1998. Correction for partial volume effects in PET: principle and validation. Journal of Nuclear Medicine, 39(5):904-911. 
Rubinski, A., D. Tosun, N. Franzmeier, J. Neitzel, L. Frontzkowski, M. Weiner, and M. Ewers 2021. Lower cerebral perfusion is associated with tau-PET in the entorhinal cortex across the Alzheimer's continuum. Neurobiology of Aging, 102:111-118.

Sanchez, J. S., J. A. Becker, H. I. L. Jacobs, B. J. Hanseeuw, S. Jiang, A. P. Schultz, M. J. Properzi, S. R. Katz, A. Beiser, C. L. Satizabal, A. O’Donnell, C. DeCarli, R. Killiany, G. El Fakhri, M. D. Normandin, T. Gómez-Isla, Y. T. Quiroz, D. M. Rentz, R. A. Sperling, S. Seshadri, J. Augustinack, J. C. Price, and K. A. Johnson 2021. The cortical origin and initial spread of medial temporal tauopathy in Alzheimer's disease assessed with positron emission tomography. Science Translational Medicine, 13(577):eabc0655.

Schreiber, S., S. M. Landau, A. Fero, F. Schreiber, and W. J. Jagust 2015. Comparison of visual and quantitative florbetapir F 18 positron emission tomography analysis in predicting mild cognitive impairment outcomes. JAMA Neurology, 72(10):1183-1190.

Scutari, M. 2010. Learning Bayesian Networks wirth bnlearn R package. Journal of Statistical Software, 35(3).

Sokoloff, L. 1993. Sites and mechanisms of function-related changes in energy metabolism in the nervous system. Developmental Neuroscience, 15(3-5):194-206.

Sperling, R. A., P. S. Aisen, L. A. Beckett, D. A. Bennett, S. Craft, A. M. Fagan, T. Iwatsubo, C. R. Jack, J. Kaye, T. J. Montine, D. C. Park, E. M. Reiman, C. C. Rowe, E. Siemers, Y. Stern, K. Yaffe, M. C. Carrillo, B. Thies, M. Morrison-Bogorad, M. V. Wagster, and C. H. Phelps 2011. Toward defining the preclinical stages of Alzheimer's disease: recommendations from the National Institute on Aging-Alzheimer's Association workgroups on diagnostic guidelines for Alzheimer's disease. Alzheimer's 83 Dementia, 7(3):280-292.

Strom, A., L. Iaccarino, L. Edwards, O. H. Lesman-Segev, D. N. Soleimani-Meigooni, J. Pham, S. L. Baker, S. Landau, W. J. Jagust, B. L. Miller, H. J. Rosen, M. L. Gorno-Tempini, G. D. Rabinovici, R. La Joie, and Alzheimer's Disease Neuroimaging Initiative 2021. Glucose metabolism reflects local atrophy and tau pathology in symptomatic Alzheimer's disease. medRxiv.

Teipel, S. and M. J. Grothe 2016. Does posterior cingulate hypometabolism result from disconnection or local pathology across preclinical and clinical stages of Alzheimer's disease? European Journal of Nuclear Medicine and Molecular Imaging, 43(3):526-536.

Thomas, B. A., K. Erlandsson, M. Modat, L. Thurfjell, R. Vandenberghe, S. Ourselin, and B. F. Hutton 2011. The importance of appropriate partial volume correction for PET quantification in Alzheimer's disease. European Journal of Nuclear Medicine and Molecular Imaging, 38(6):1104-1119.

Tingley, D., T. Yamamoto, K. Hirose, L. Keele, and K. Imai 2014. mediation: R Package for Causal Mediation Analysis. Journal of Statistical Software, 59(5).

Villemagne, V. L., S. Burnham, P. Bourgeat, B. Brown, K. A. Ellis, O. Salvado, C. Szoeke, S. L. Macaulay, R. Martins, P. Maruff, D. Ames, C. C. Rowe, and C. L. Masters 2013. Amyloid $\beta$ deposition, neurodegeneration, and cognitive decline in sporadic Alzheimer's disease: a prospective cohort study. Lancet Neurology, 12(4):357-367.

Wickham, H., M. Averick, J. Bryan, W. Chang, L. McGowan, R. François, G. Grolemund, A. Hayes, L. Henry, J. Hester, M. Kuhn, T. Pedersen, E. Miller, S. Bache, K. Müller, J. Ooms, D. Robinson, D. Seidel, V. Spinu, K. Takahashi, D. Vaughan, C. Wilke, K. Woo, and H. Yutani 2019. Welcome to the Tidyverse. Journal of Open Source Software, 4(43):1686.

Wu, J., S. Hussaini, and I. Bastille 2016. Neuronal activity enhances tau propagation and tau pathology in vivo. Nat Neurosci, 19(8):1085-1092.

Xie, Y. 2018. knitr: A general-purpose package for dynamic report generation in R.

Zhou, Y., S. M. Resnick, W. Ye, H. Fan, D. P. Holt, W. E. Klunk, C. A. Mathis, R. Dannals, and D. F. Wong 2007. Using a reference tissue model with spatial constraint to quantify [11C]Pittsburgh compound B PET for early diagnosis of Alzheimer's disease. NeuroImage, 36(2):298-312. 
medRxiv preprint doi: https://doi.org/10.1101/2021.07.01.21259866; this version posted July 6, 2021. The copyright holder for this preprint (which was not certified by peer review) is the author/funder, who has granted medRxiv a license to display the preprint in perpetuity.

This article is a US Government work. It is not subject to copyright under 17 USC 105 and is also made available for use under a CCO license.

Ziontz, J., M. Bilgel, A. T. Shafer, A. Moghekar, W. Elkins, J. Helphrey, G. Gomez, D. June, M. A. McDonald, R. F. Dannals, B. B. Azad, L. Ferrucci, D. F. Wong, and S. M. Resnick 2019. Tau pathology in cognitively normal older adults. Alzheimer's \& Dementia: Diagnosis, Assessment $\& 3$ Disease Monitoring, 11:637-645. 


\section{Supplementary Material}

\section{A Conditional independencies for more parsimonious DAGs}

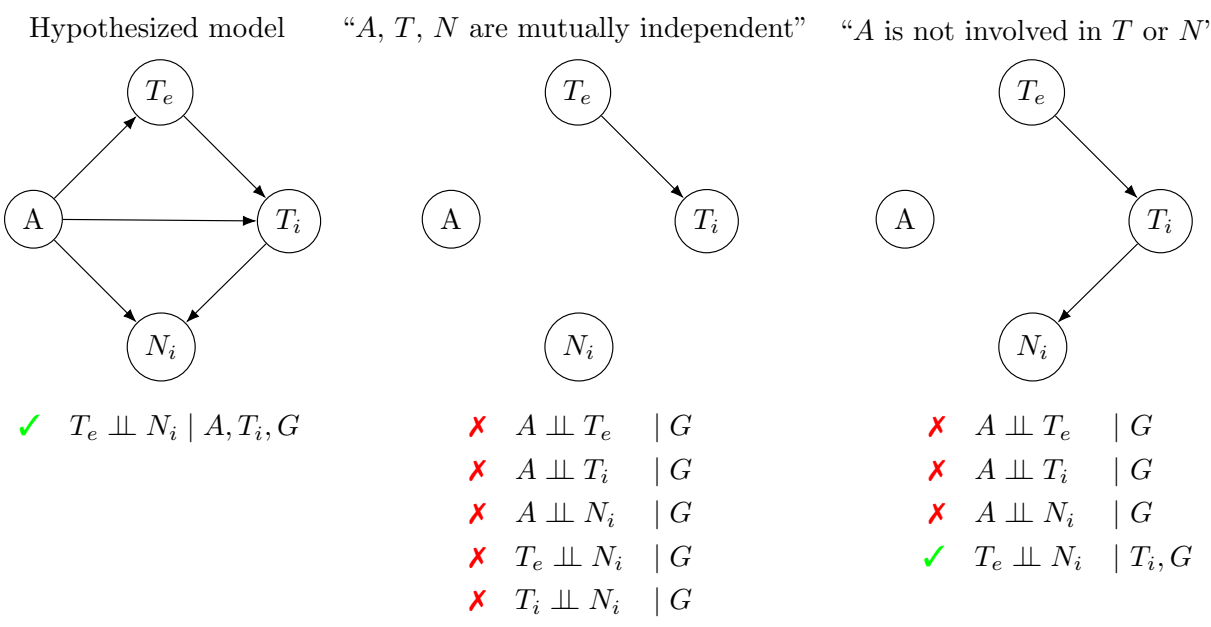

" $A$ is not involved in $T$ " "Neither $A$ nor $T$ is involved in $N " \quad$ " $A$ is not involved in $N$ "
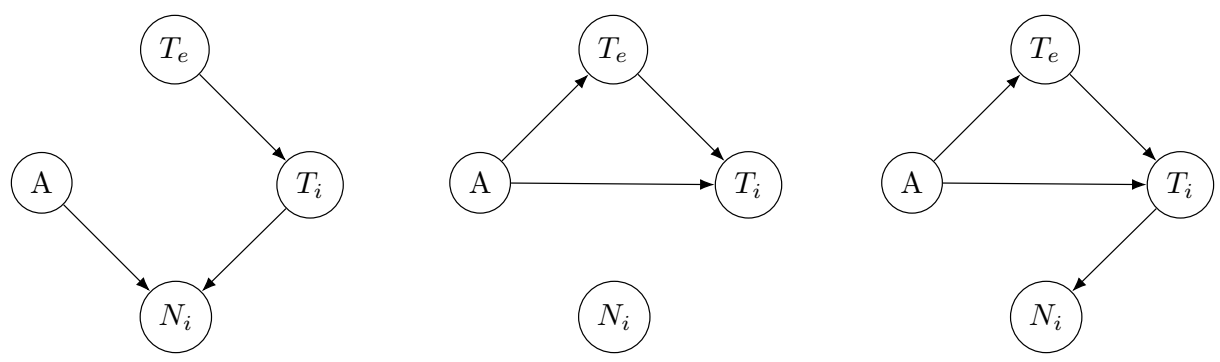

$$
\begin{array}{lll}
\times & A \Perp T_{e} & \mid G \\
\times & A \Perp T_{i} & \mid G \\
\checkmark & T_{e} \Perp N_{i} & \mid T_{i}, G
\end{array}
$$$$
\times \quad A \Perp N_{i} \quad \mid G
$$

$\times \quad T_{e} \Perp N_{i} \mid G$

× $A \Perp N_{i} \quad \mid T_{i}, G$

× $\quad T_{i} \Perp N_{i} \mid G$

$\checkmark \quad T_{e} \Perp N_{i} \quad \mid T_{i}, G$

" $A$ is not involved in MTL $T$ "

" $A$ is not involved in neocortical $T$ "

" $T$ is not involved in $N$ "
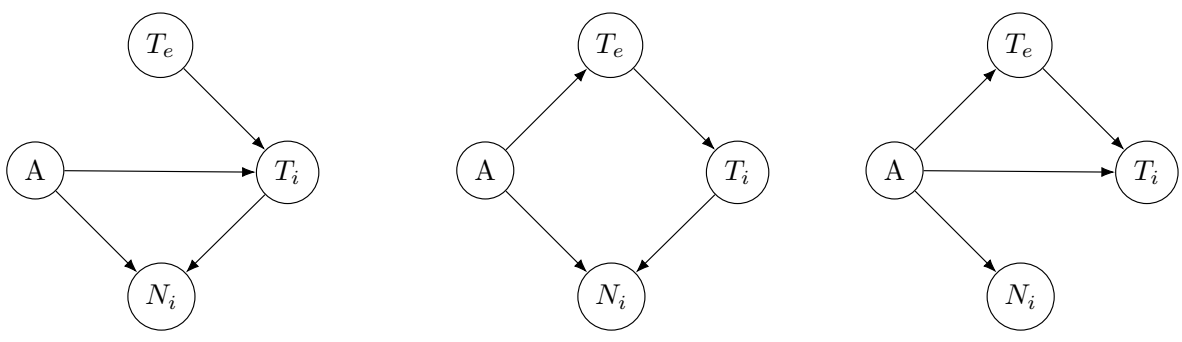

$$
\begin{array}{llll}
x & A \Perp T_{e} & \mid G \\
\checkmark & T_{e} \Perp N_{i} & A, T_{i}, G
\end{array}
$$

$$
\begin{array}{lll}
x & A \Perp T_{i} & \mid T_{e}, G \\
\checkmark & T_{e} \Perp N_{i} & \mid A, T_{i}, G
\end{array}
$$

$$
\begin{array}{ll|l}
\checkmark & T_{e} \Perp N_{i} & \mid A, G \\
\times & T_{i} \Perp N_{i} & A, G
\end{array}
$$

Figure S1. DAGs investigated in the BLSA. $G$ indicates the collection of covariates (age, sex, education, and $A P O E$ $\varepsilon 4$ status). Each of these covariates is a node with an arrow towards each of the four nodes shown in this diagram (nodes for covariates are not shown). The conditional independencies implied by each DAG are listed below the graphs. $\checkmark$ indicates that the partial correlation is not statistically significant in the observed data (and therefore, the DAG is not inconsistent with the data). $x$ indicates that the partial correlation is statistically significant (and therefore, the DAG is inconsistent with the data). $A=$ binary amyloid status, $T_{e}=\mathrm{EC}$ tau, $T_{i}=\mathrm{ITG}$ tau, $N_{i}=$ ITG CBF. 
medRxiv preprint doi: https://doi.org/10.1101/2021.07.01.21259866; this version posted July 6, 2021. The copyright holder for this preprint (which was not certified by peer review) is the author/funder, who has granted medRxiv a license to display the preprint in perpetuity.

This article is a US Government work. It is not subject to copyright under 17 USC 105 and is also made available for use under a CCO license.

\section{B Cross-sectional associations}

Table S1. Linear regression models investigating the cross-sectional association between amyloid group and entorhinal cortex FTP SUVR $z$-score in the BLSA (left) and ADNI (right).

Dependent variable:

Entorhinal cortex FTP SUVR $z$-score

\begin{tabular}{|c|c|c|}
\hline & \\
\hline & BLSA & ADNI \\
\hline Intercept & $\begin{array}{c}-0.431^{*}(0.181) \\
\mathrm{p}=0.020\end{array}$ & $\begin{array}{c}-0.439^{*}(0.176) \\
\mathrm{p}=0.014\end{array}$ \\
\hline Age at FTP PET scan & $\begin{array}{c}0.015(0.011) \\
\mathrm{p}=0.194\end{array}$ & $\begin{array}{c}0.027^{*}(0.012) \\
\mathrm{p}=0.030\end{array}$ \\
\hline $\begin{array}{l}\text { Sex } \\
(\text { ref = female })\end{array}$ & $\begin{array}{c}0.104(0.194) \\
p=0.594\end{array}$ & $\begin{array}{c}-0.213(0.178) \\
\mathrm{p}=0.235\end{array}$ \\
\hline Years of education & $\begin{array}{c}0.018(0.046) \\
\mathrm{p}=0.696\end{array}$ & $\begin{array}{c}0.005(0.031) \\
\mathrm{p}=0.877\end{array}$ \\
\hline $\begin{array}{l}A P O E \text { status } \\
\left(\text { ref }=\varepsilon 4^{-}\right)\end{array}$ & $\begin{array}{c}0.087(0.207) \\
p=0.678\end{array}$ & $\begin{array}{c}0.541^{* *}(0.198) \\
\mathrm{p}=0.008\end{array}$ \\
\hline $\begin{array}{l}\text { Amyloid group } \\
\left(\text { ref }=A^{-}\right)\end{array}$ & $\begin{array}{c}0.730^{* * *}(0.212) \\
\mathrm{p}=0.001\end{array}$ & $\begin{array}{c}0.443^{*}(0.191) \\
\mathrm{p}=0.023\end{array}$ \\
\hline
\end{tabular}


Table S2. Linear regression models investigating the cross-sectional association of amyloid group entorhinal cortex FTP SUVR $z$-score with ITG FTP SUVR $z$-score in the BLSA (left) and ADNI (right).

Dependent variable:

ITG FTP SUVR $z$-score

\begin{tabular}{|c|c|c|}
\hline & \multirow{2}{*}{\multicolumn{2}{|c|}{ BLSA }} \\
\hline & & \\
\hline Intercept & $\begin{array}{c}-0.334^{* *}(0.116) \\
\mathrm{p}=0.005\end{array}$ & $\begin{array}{c}-0.248(0.128) \\
\mathrm{p}=0.056\end{array}$ \\
\hline Age at FTP PET scan & $\begin{array}{c}-0.012(0.007) \\
\mathrm{p}=0.102\end{array}$ & $\begin{array}{c}-0.006(0.009) \\
\mathrm{p}=0.476\end{array}$ \\
\hline $\begin{array}{l}\text { Sex } \\
(\text { ref = female })\end{array}$ & $\begin{array}{l}0.333^{* *}(0.120) \\
\mathrm{p}=0.007\end{array}$ & $\begin{array}{c}0.066(0.120) \\
\mathrm{p}=0.585\end{array}$ \\
\hline Years of education & $\begin{array}{c}0.051(0.029) \\
\mathrm{p}=0.077\end{array}$ & $\begin{array}{c}-0.016(0.021) \\
\mathrm{p}=0.439\end{array}$ \\
\hline $\begin{array}{l}A P O E \text { status } \\
\left(\text { ref }=\varepsilon 4^{-}\right)\end{array}$ & $\begin{array}{c}-0.028(0.128) \\
\mathrm{p}=0.829\end{array}$ & $\begin{array}{c}-0.178(0.137) \\
\mathrm{p}=0.198\end{array}$ \\
\hline $\begin{array}{l}\text { Amyloid group } \\
\left(\mathrm{ref}=\mathrm{A}^{-}\right)\end{array}$ & $\begin{array}{l}0.417^{* *}(0.138) \\
\mathrm{p}=0.004\end{array}$ & $\begin{array}{c}0.406^{* *}(0.133) \\
\mathrm{p}=0.003\end{array}$ \\
\hline EC FTP SUVR $z$-score & $\begin{array}{c}0.383^{* * *}(0.097) \\
\mathrm{p}=0.0002\end{array}$ & $\begin{array}{c}0.244^{*}(0.117) \\
\mathrm{p}=0.041\end{array}$ \\
\hline $\begin{array}{l}\text { Amyloid group } \\
\times \text { EC FTP SUVR } z \text {-score }\end{array}$ & $\begin{array}{l}0.486^{* * *}(0.126) \\
\mathrm{p}=0.0003\end{array}$ & $\begin{array}{c}0.658^{* * *}(0.137) \\
\mathrm{p}=0.000005\end{array}$ \\
\hline Note: & & .00 \\
\hline
\end{tabular}


Table S3. Linear regression models investigating the cross-sectional association of amyloid group ITG FTP SUVR $z$-score with ITG PiB $R_{1} z$-score in the BLSA (left) and ITG FDG SUVR $z$-score in the ADNI (right).

Dependent variable:

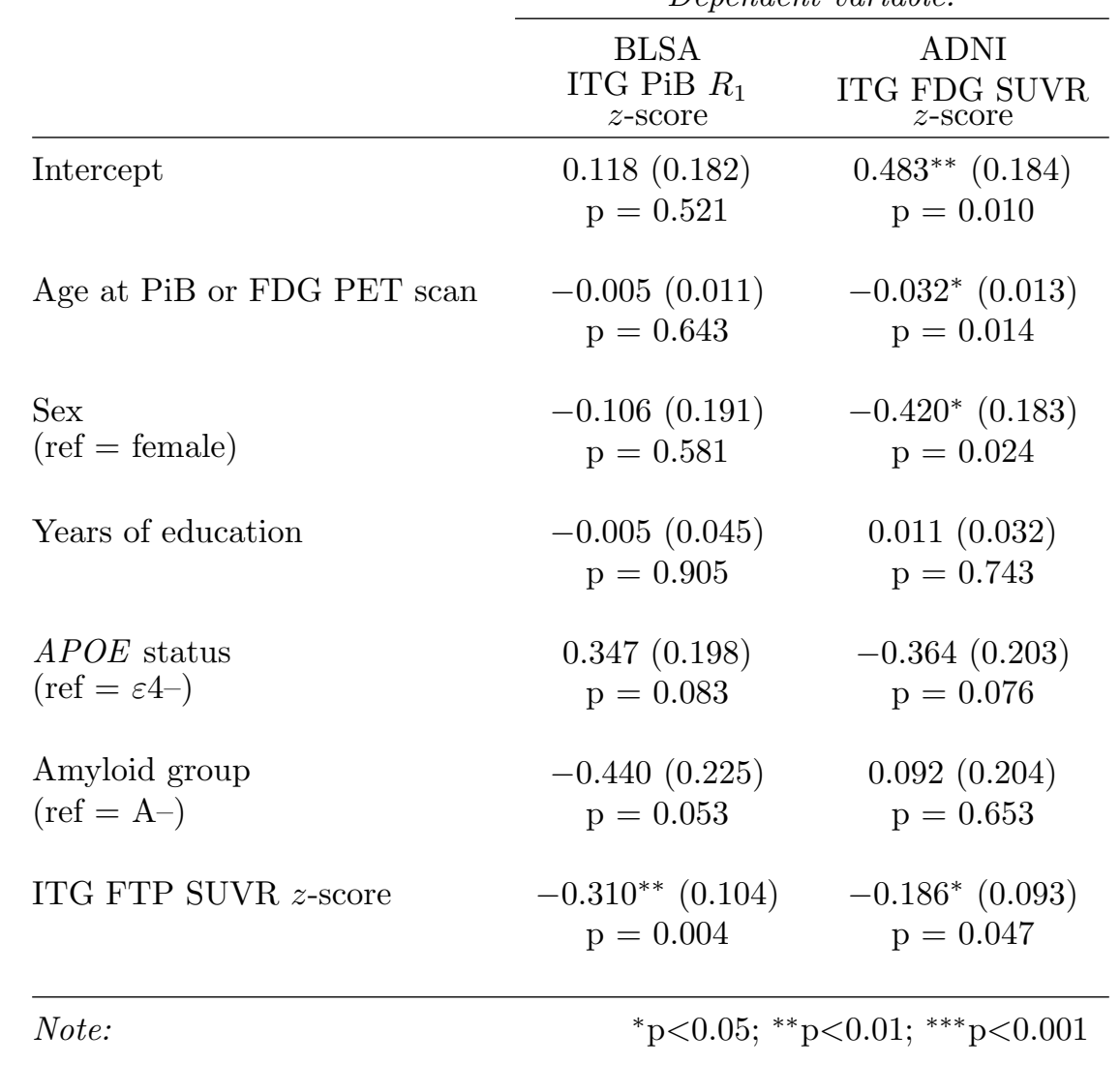


Table S4. Linear regression models investigating the cross-sectional association of amyloid group ITG FTP SUVR $z$-score with ITG ICV-adjusted volume residual $z$-score in the BLSA (left) and ADNI (right).

\section{Dependent variable:}

\begin{tabular}{lcc} 
& \multicolumn{2}{c}{ ITG volume (ICV-adj. residual) $z$-score } \\
\cline { 2 - 3 } & $0.342(0.193)$ & $0.347^{*}(0.173)$ \\
\hline Intercept & $\mathrm{p}=0.080$ & $\mathrm{p}=0.048$ \\
& $-0.035^{* *}(0.012)$ & $-0.039^{* *}(0.012)$ \\
Age at MRI scan & $\mathrm{p}=0.004$ & $\mathrm{p}=0.002$ \\
& $0.128(0.202)$ & $-0.196(0.171)$ \\
Sex & $\mathrm{p}=0.526$ & $\mathrm{p}=0.253$ \\
(ref $=$ female) & $0.003(0.047)$ & $0.008(0.030)$ \\
Years of education & $\mathrm{p}=0.946$ & $\mathrm{p}=0.800$ \\
& $-0.388(0.210)$ & $0.316(0.190)$ \\
APOE status & $\mathrm{p}=0.068$ & $\mathrm{p}=0.100$ \\
(ref $=$ \&4-) & $-0.118(0.238)$ & $-0.267(0.191)$ \\
Amyloid group & $\mathrm{p}=0.621$ & $\mathrm{p}=0.165$ \\
(ref $=$ A-) & $-0.146(0.110)$ & $-0.370^{* * *}(0.087)$ \\
ITG FTP SUVR $z$-score & $\mathrm{p}=0.185$ & $\mathrm{p}=0.00004$ \\
& & \\
\hline Note: & ${ }^{*} \mathrm{p}<0.05 ;{ }^{* *} \mathrm{p}<0.01 ;{ }^{* * *} \mathrm{p}<0.001$ \\
& &
\end{tabular}


medRxiv preprint doi: https://doi.org/10.1101/2021.07.01.21259866; this version posted July 6, 2021. The copyright holder for this preprint (which was not certified by peer review) is the author/funder, who has granted medRxiv a license to display the preprint in perpetuity.

This article is a US Government work. It is not subject to copyright under 17 USC 105 and is also made available for use under a CCO license.

\section{Causal mediation analysis}

\section{Sensitivity analysis}

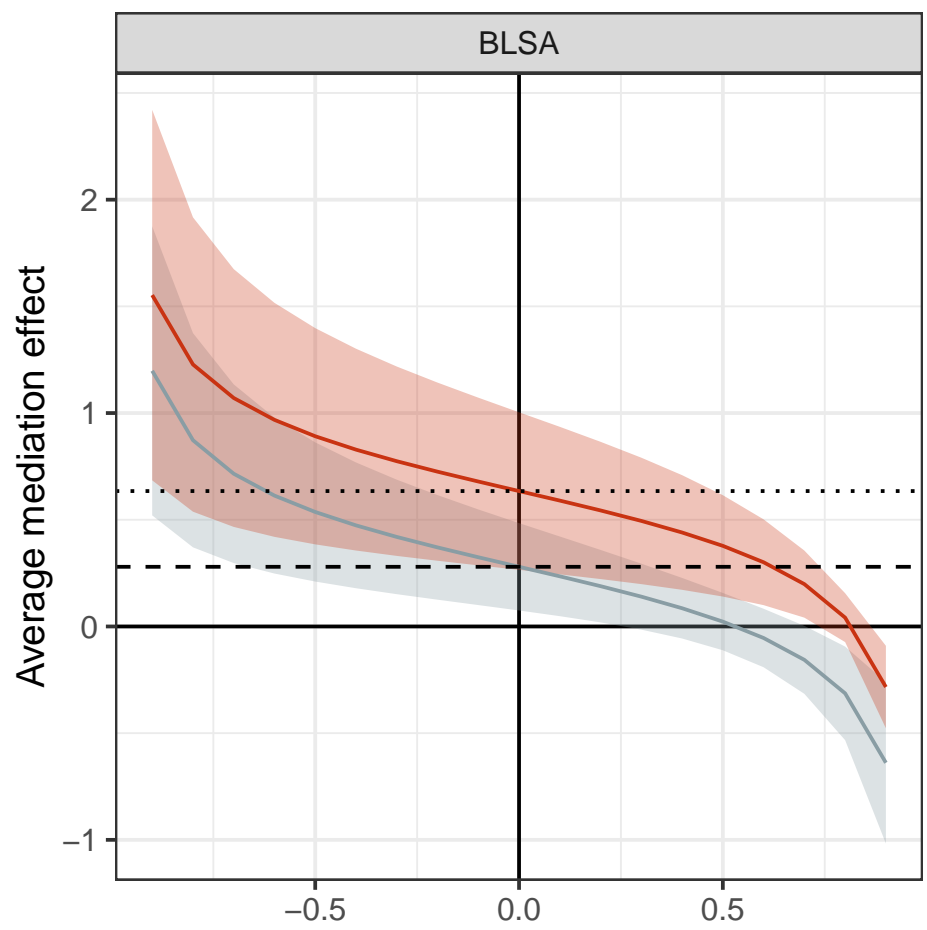

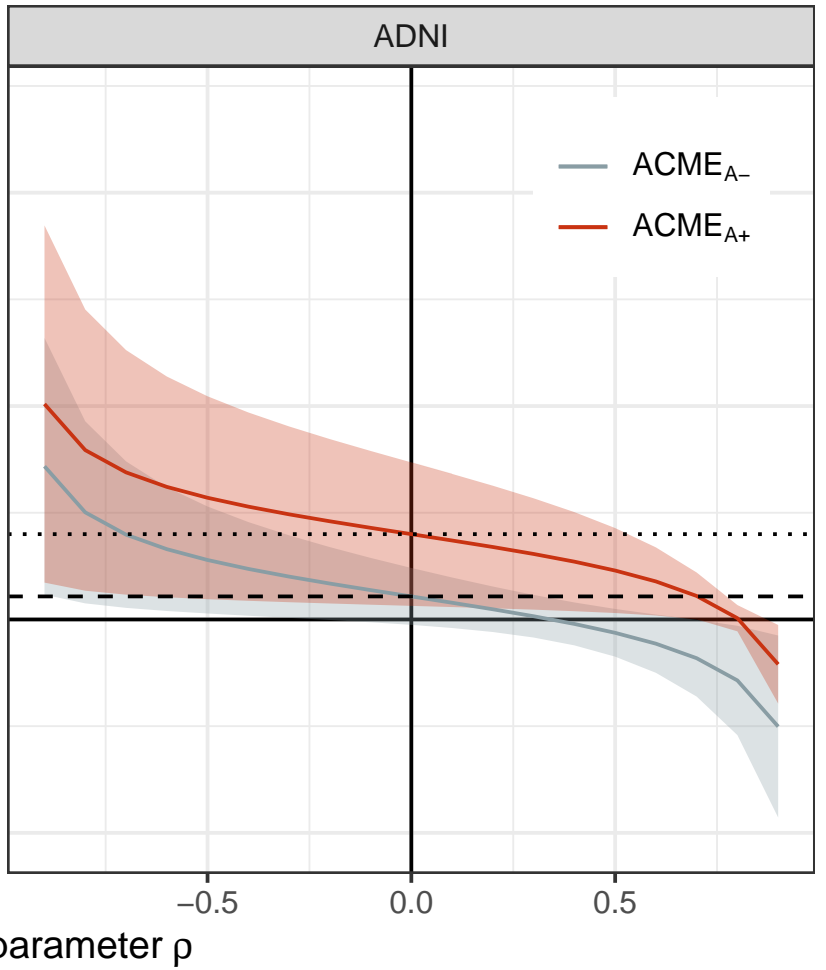

Figure S2. Sensitivity analyses for the mediation models investigating entorhinal cortex tau as a mediator of the association between amyloid group and inferior temporal gyrus tau. 
medRxiv preprint doi: https://doi.org/10.1101/2021.07.01.21259866; this version posted July 6, 2021. The copyright holder for this preprint (which was not certified by peer review) is the author/funder, who has granted medRxiv a license to display the preprint in perpetuity.

This article is a US Government work. It is not subject to copyright under 17 USC 105 and is also made available for use under a CCO license.

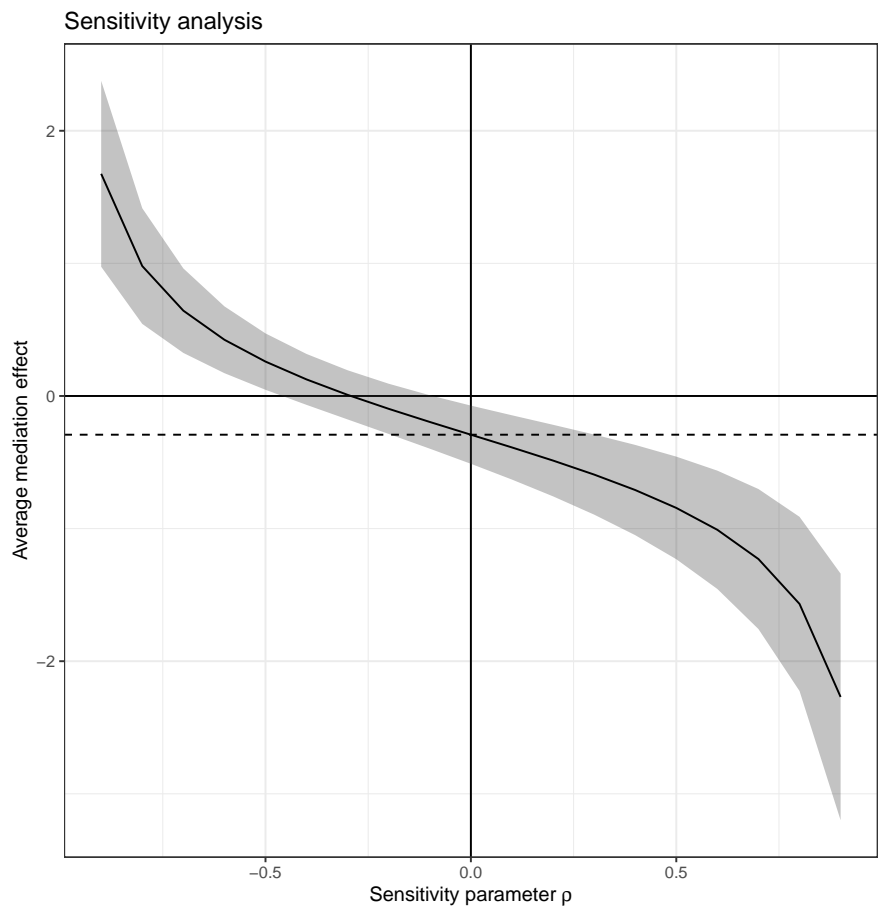

Figure S3. Sensitivity analysis for the mediation model investigating inferior temporal gyrus tau as a mediator of the association between amyloid group and inferior temporal gyrus cerebral blood flow in the BLSA.

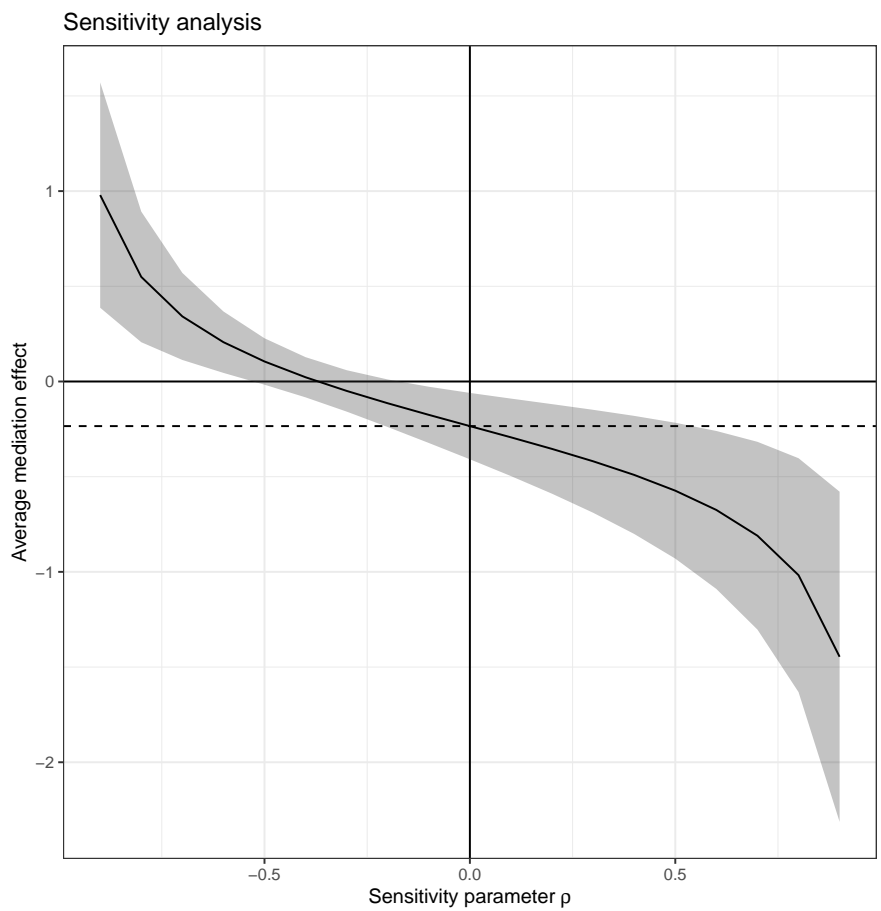

Figure S4. Sensitivity analysis for the mediation model investigating inferior temporal gyrus tau as a mediator of the association between amyloid group and inferior temporal gyrus volume in the ADNI. 


\section{Longitudinal analysis}

Table S5. Linear mixed effects models investigating the association between rate of change in entorhinal cortex FTP SUVR $z$-score and baseline amyloid group in the BLSA (left) and ADNI (right).

Dependent variable:

\begin{tabular}{|c|c|c|}
\hline & Entorhinal corte & TP SUVR $z$-score \\
\hline & BLSA & ADNI \\
\hline Intercept & $\begin{array}{c}-0.403^{*}(0.179) \\
\mathrm{p}=0.025\end{array}$ & $\begin{array}{c}-0.436^{*}(0.176) \\
\mathrm{p}=0.014\end{array}$ \\
\hline Age at baseline FTP PET scan & $\begin{array}{c}0.014(0.011) \\
\mathrm{p}=0.212\end{array}$ & $\begin{array}{c}0.027^{*}(0.012) \\
\mathrm{p}=0.031\end{array}$ \\
\hline $\begin{array}{l}\text { Sex } \\
(\text { ref }=\text { female })\end{array}$ & $\begin{array}{c}0.096(0.191) \\
p=0.614\end{array}$ & $\begin{array}{c}-0.214(0.178) \\
\mathrm{p}=0.231\end{array}$ \\
\hline Years of education & $\begin{array}{c}0.012(0.046) \\
\mathrm{p}=0.792\end{array}$ & $\begin{array}{c}0.006(0.031) \\
\mathrm{p}=0.841\end{array}$ \\
\hline $\begin{array}{l}A P O E \text { status } \\
\left(\text { ref }=\varepsilon 4^{-}\right)\end{array}$ & $\begin{array}{c}0.071(0.204) \\
p=0.729\end{array}$ & $\begin{array}{c}0.538^{* *}(0.198) \\
\mathrm{p}=0.007\end{array}$ \\
\hline $\begin{array}{l}\text { Amyloid group } \\
\left(\text { ref }=\mathrm{A}^{-}\right)\end{array}$ & $\begin{array}{c}0.715^{* * *}(0.210) \\
\mathrm{p}=0.001\end{array}$ & $\begin{array}{c}0.432^{*}(0.192) \\
\mathrm{p}=0.025\end{array}$ \\
\hline Time from baseline & $\begin{array}{c}-0.035(0.035) \\
\mathrm{p}=0.324\end{array}$ & $\begin{array}{c}-0.008(0.022) \\
p=0.713\end{array}$ \\
\hline $\begin{array}{l}\text { Amyloid group } \\
\times \text { time }\end{array}$ & $\begin{array}{c}0.079(0.061) \\
p=0.193\end{array}$ & $\begin{array}{c}0.068^{*}(0.033) \\
\mathrm{p}=0.040\end{array}$ \\
\hline
\end{tabular}


Table S6. Linear mixed effects models investigating the association of baseline amyloid group and EC FTP SUVR $z$-score with rate of change in ITG FTP SUVR $z$-score in the BLSA (left) and ADNI (right).

\begin{tabular}{|c|c|c|}
\hline & \multicolumn{2}{|c|}{$\begin{array}{l}\text { Dependent variable: } \\
\text { ITG FTP SUVR } z \text {-score }\end{array}$} \\
\hline & BLSA & ADNI \\
\hline Intercept & $\begin{array}{c}-0.320^{* *}(0.114) \\
\mathrm{p}=0.005\end{array}$ & $\begin{array}{c}-0.240(0.132) \\
\mathrm{p}=0.070\end{array}$ \\
\hline Age at baseline FTP PET scan & $\begin{array}{c}-0.012(0.007) \\
\mathrm{p}=0.079\end{array}$ & $\begin{array}{c}-0.007(0.009) \\
p=0.434\end{array}$ \\
\hline $\begin{array}{l}\text { Sex } \\
(\text { ref }=\text { female })\end{array}$ & $\begin{array}{c}0.342^{* *}(0.118) \\
\mathrm{p}=0.004\end{array}$ & $\begin{array}{c}0.054(0.123) \\
\mathrm{p}=0.661\end{array}$ \\
\hline Years of education & $\begin{array}{c}0.051(0.028) \\
\mathrm{p}=0.068\end{array}$ & $\begin{array}{c}-0.020(0.022) \\
\mathrm{p}=0.349\end{array}$ \\
\hline $\begin{array}{l}A P O E \text { status } \\
\left(\text { ref }=\varepsilon 4^{-}\right)\end{array}$ & $\begin{array}{c}-0.035(0.125) \\
\mathrm{p}=0.780\end{array}$ & $\begin{array}{c}-0.167(0.140) \\
\mathrm{p}=0.233\end{array}$ \\
\hline $\begin{array}{l}\text { Amyloid group } \\
\left(\text { ref }=A^{-}\right)\end{array}$ & $\begin{array}{c}0.393^{* *}(0.137) \\
\mathrm{p}=0.005\end{array}$ & $\begin{array}{c}0.393^{* *}(0.137) \\
\mathrm{p}=0.004\end{array}$ \\
\hline Baseline EC FTP SUVR $z$-score & $\begin{array}{c}0.393^{* * *}(0.096) \\
\mathrm{p}=0.00004\end{array}$ & $\begin{array}{c}0.233(0.121) \\
\mathrm{p}=0.054\end{array}$ \\
\hline $\begin{array}{l}\text { Amyloid group } \\
\times \text { EC FTP SUVR } z \text {-score }\end{array}$ & $\begin{array}{c}0.473^{* * *}(0.124) \\
p=0.0002\end{array}$ & $\begin{array}{c}0.672^{* * *}(0.141) \\
\mathrm{p}=0.000002\end{array}$ \\
\hline Time from baseline & $\begin{array}{c}0.034(0.023) \\
\mathrm{p}=0.143\end{array}$ & $\begin{array}{c}0.053^{*}(0.023) \\
\mathrm{p}=0.020\end{array}$ \\
\hline $\begin{array}{l}\text { Amyloid group } \\
\times \text { time }\end{array}$ & $\begin{array}{c}0.043(0.039) \\
\mathrm{p}=0.275\end{array}$ & $\begin{array}{c}0.054(0.030) \\
\mathrm{p}=0.073\end{array}$ \\
\hline $\begin{array}{l}\text { EC FTP SUVR } z \text {-score } \\
\times \text { time }\end{array}$ & $\begin{array}{c}-0.030(0.027) \\
\mathrm{p}=0.277\end{array}$ & $\begin{array}{c}0.080^{* * *}(0.020) \\
\mathrm{p}=0.0001\end{array}$ \\
\hline
\end{tabular}


Table S7. Linear mixed effects models investigating the association of baseline amyloid group and ITG FTP SUVR $z$-score with rate of change in ITG PiB $R_{1} z$-score in the BLSA (left) and rate of change in ITG FDG SUVR $z$-score in the ADNI (right).

\begin{tabular}{|c|c|c|}
\hline & \multicolumn{2}{|c|}{ Dependent variable: } \\
\hline & $\begin{array}{c}\text { BLSA } \\
\text { ITG PiB } R_{1} \\
z \text {-score }\end{array}$ & $\begin{array}{c}\text { ADNI } \\
\text { ITG FDG SUVR } \\
z \text {-score }\end{array}$ \\
\hline Intercept & $\begin{array}{c}0.149(0.176) \\
\mathrm{p}=0.396\end{array}$ & $\begin{array}{c}0.398^{*}(0.172) \\
\mathrm{p}=0.021\end{array}$ \\
\hline Age at baseline FTP PET scan & $\begin{array}{c}-0.003(0.011) \\
\mathrm{p}=0.743\end{array}$ & $\begin{array}{c}-0.031^{* *}(0.012) \\
\mathrm{p}=0.010\end{array}$ \\
\hline $\begin{array}{l}\text { Sex } \\
(\text { ref = female })\end{array}$ & $\begin{array}{c}-0.183(0.184) \\
p=0.320\end{array}$ & $\begin{array}{c}-0.299(0.168) \\
p=0.075\end{array}$ \\
\hline Years of education & $\begin{array}{c}0.004(0.043) \\
\mathrm{p}=0.920\end{array}$ & $\begin{array}{c}0.010(0.029) \\
\mathrm{p}=0.729\end{array}$ \\
\hline $\begin{array}{l}A P O E \text { status } \\
\left(\mathrm{ref}=\varepsilon 4^{-}\right)\end{array}$ & $\begin{array}{c}0.288(0.191) \\
\mathrm{p}=0.132\end{array}$ & $\begin{array}{c}-0.333(0.186) \\
\mathrm{p}=0.074\end{array}$ \\
\hline $\begin{array}{l}\text { Amyloid group } \\
\left(\text { ref }=\mathrm{A}^{-}\right)\end{array}$ & $\begin{array}{c}-0.385(0.217) \\
\mathrm{p}=0.077\end{array}$ & $\begin{array}{c}0.102(0.193) \\
\mathrm{p}=0.598\end{array}$ \\
\hline Baseline ITG FTP SUVR $z$-score & $\begin{array}{c}-0.311^{* *}(0.100) \\
\mathrm{p}=0.002\end{array}$ & $\begin{array}{c}-0.191^{*}(0.088) \\
\mathrm{p}=0.031\end{array}$ \\
\hline Time from baseline & $\begin{array}{c}-0.033^{*}(0.017) \\
\mathrm{p}=0.050\end{array}$ & $\begin{array}{c}-0.068^{*}(0.028) \\
\mathrm{p}=0.016\end{array}$ \\
\hline $\begin{array}{l}\text { Amyloid group } \\
\times \text { time }\end{array}$ & $\begin{array}{c}0.014(0.031) \\
\mathrm{p}=0.644\end{array}$ & $\begin{array}{c}-0.007(0.040) \\
\mathrm{p}=0.867\end{array}$ \\
\hline $\begin{array}{l}\text { ITG FTP SUVR } z \text {-score } \\
\times \text { time }\end{array}$ & $\begin{array}{c}0.029(0.019) \\
\mathrm{p}=0.128\end{array}$ & $\begin{array}{c}-0.090^{*}(0.040) \\
\mathrm{p}=0.024\end{array}$ \\
\hline
\end{tabular}


Table S8. Linear mixed effects models investigating the association of baseline amyloid group and ITG FTP SUVR $z$-score with rate of change in ITG ICV-adjusted volume residual $z$-score in the BLSA (left) and ADNI (right).

\section{Dependent variable:}

\begin{tabular}{|c|c|c|}
\hline & ITG volume $(\mathrm{ICV}$ & j. residual) $z$-score \\
\hline & BLSA & ADNI \\
\hline Intercept & $0.302(0.184)$ & $0.354^{*}(0.172)$ \\
\hline & $\mathrm{p}=0.101$ & $\mathrm{p}=0.041$ \\
\hline Age at baseline FTP PET scan & $-0.037^{* * *}(0.011)$ & $-0.041^{* * *}(0.012)$ \\
\hline & $\mathrm{p}=0.001$ & $\mathrm{p}=0.0005$ \\
\hline Sex & $0.133(0.192)$ & $-0.169(0.169)$ \\
\hline$($ ref $=$ female $)$ & $\mathrm{p}=0.490$ & $\mathrm{p}=0.318$ \\
\hline Years of education & $0.003(0.045)$ & $0.014(0.030)$ \\
\hline & $\mathrm{p}=0.956$ & $\mathrm{p}=0.624$ \\
\hline$A P O E$ status & $-0.351(0.200)$ & $0.234(0.188)$ \\
\hline$\left(\mathrm{ref}=\varepsilon 4^{-}\right)$ & $\mathrm{p}=0.080$ & $\mathrm{p}=0.214$ \\
\hline Amyloid group & $-0.035(0.227)$ & $-0.232(0.190)$ \\
\hline$\left(\mathrm{ref}=\mathrm{A}^{-}\right)$ & $\mathrm{p}=0.877$ & $\mathrm{p}=0.223$ \\
\hline Baseline ITG FTP SUVR $z$-score & $-0.160(0.105)$ & $-0.366^{* * *}(0.087)$ \\
\hline & $\mathrm{p}=0.127$ & $\mathrm{p}=0.00003$ \\
\hline Time from baseline & $-0.088^{* * *}(0.012)$ & $-0.068^{* *}(0.024)$ \\
\hline & $\mathrm{p}=0.000$ & $\mathrm{p}=0.005$ \\
\hline Amyloid group & $-0.013(0.023)$ & $-0.002(0.041)$ \\
\hline$\times$ time & $\mathrm{p}=0.571$ & $\mathrm{p}=0.958$ \\
\hline ITG FTP SUVR $z$-score & $-0.049^{* * *}(0.014)$ & $-0.064^{* *}(0.024)$ \\
\hline$\times$ time & $\mathrm{p}=0.0005$ & $\mathrm{p}=0.007$ \\
\hline
\end{tabular}

